\title{
ON THE EXISTENCE OF A UNIVERSAL GERM OF DEFORMATIONS FOR ELLIPTIC PSEUDOGROUP STRUCTURES ON COMPACT MANIFOLDS
}

\author{
BY
}

\author{
SURESH H. MOOLGAVKAR(1)
}

\begin{abstract}
The purpose of this paper is to prove the existence of a versal germ of deformations for elliptic pseudogroup structures on compact manifolds. Under suitable restrictions, the versal germ is shown to be universal.
\end{abstract}

Introduction. Kodaira and Spencer initiated the theory of deformations of compact complex manifolds in 1958 [7], [8]. The existence problem was solved soon after by Kodaira, Nirenberg and Spencer [8], [9], under the assumption that the cohomology group $H^{2}(M, \theta)=0$, where $\theta=$ sheaf of germs of infinitesimal automorphisms of the complex structure i.e., $\theta=$ sheaf of germs of holomorphic vector fields on $M$. The parameter space for deformations, in the Kodaira Spencer theory, is an open neighborhood of zero in $H^{1}(M, \theta)$.

In 1962, Kuranishi succeeded in removing the restriction $H^{2}(M, \theta)=0$, but only at the cost of admitting an analytic variety in $H^{1}(M, \theta)$ as parameter space. That is, Kuranishi proved the existence of a versal (complete) germ of deformations of an arbitrary compact complex manifold. He presented a new and simplified proof for this theorem at the Minnesota Conference in 1964 [12]. A detailed version of the new proof with some improvements has appeared in [13].

In the meantime, Spencer in his fundamental papers [20], [21] laid the foundations for a general theory of deformations. The existence theorem (for elliptic transitive pseudogroups) generalizing the Newlander-Nirenberg theorem for complex structures has recently been proved by Malgrange[15].

The aim of this paper is to show that Kuranishi's method of proof can easily be generalized in the context of Spencer's machinery to give an existence

Received by the editors August 31, 1973 and, in revised form, July 11, 1974.

AMS (MOS) subject classifications (1970). Primary 58H05; Secondary $58 \mathrm{G} 05$.

Key words and phrases. Lie equations, elliptic complexes, jet bundles.

(1)This work is part of the author's Johns Hopkins Ph.D. thesis. The author is greatly indebted to Professor D. C. Spencer for educating him in the intricacies of deformation theory. Thanks are also due to Professor J. H. Sampson for innumerable enlightening conversations. 
theorem for versal germs for elliptic pseudogroup structures on compact manifolds. In this, Malgrange's theorem plays a crucial role. Roughly speaking, the main theorem is: Let $M$ be an m-dimensional compact $C^{\infty}$ manifold without boundary. Let $\Gamma\left(\mathbf{R}^{m}\right)$ be an elliptic transitive pseudogroup on $\mathbf{R}^{m}$, and let $M_{0}$ be a $\Gamma\left(R^{m}\right)$ structure on $M$. Then, there exists a versal germ of deformations of $M_{0}$. The parameter space for the versal germ of deformations is an analytic variety in $H^{1}(M$, Inf) where Inf is the sheaf of germs of infinitesimal automorphisms of the $\Gamma\left(\mathrm{R}^{m}\right)$ structure $M_{0}$. If $H^{2}(M, \operatorname{Inf})=0$, then the parameter space is an open neighborhood of 0 in $H^{1}(M$, Inf) and the versal family is effective at the origin. We also prove in this paper that, under additional conditions, the versal family we construct is actually universal. Quê, using the machinery of groupoids, proved the existence of a versal family for analytic elliptic pseudogroups [17].

In $\S 1$, we review very briefly Spencer's machinery. A definitive version of this is now available [11], and the reader is referred to it for the details. In §2 we introduce deformations of pseudogroup structures and the notion of infinitesimal deformations. Finally, in $\S 3$, we prove the main theorems.

1. Review of the machinery. In this section we very briefly recall facts about the Spencer resolutions for a system of partial differential equations on a manifold $M$, and establish the notation that we shall use throughout the paper.

$M$ will always denote a compact, $C^{\infty}$ manifold (without boundary) of dimension $m$. Unless otherwise specified, everything will be $C^{\infty} . T$ and $T^{*}$ will denote the tangent and cotangent bundles of $M$ respectively; $J_{k} T$ will denote the $k$ th jet bundle associated to $T$. Let $R_{k} \subset J_{k} T$ be a formally integrable, regular system of Lie equations on $M$. (For the definitions see [11], [15], [23], [25].) [Let $\Gamma(M)$ be a transitive continuous Lie pseudogroup on $M$ of order $k$. Then the de- . fining equations for the $\Gamma(M)$ vector fields on $M$ are of this type [5].] Let Inf be the sheaf of solutions of the equation $R_{k}$. Spencer has constructed the complexes [11], [20], [21], [22], [25]

$$
I_{l}: 0 \rightarrow \operatorname{Inf} \stackrel{i_{l}}{\longrightarrow} C_{l}^{0} R_{k} \stackrel{D}{\longrightarrow} C_{l}^{1} R_{k} \stackrel{D}{\longrightarrow} \cdots \stackrel{D}{\longrightarrow} C_{l}^{m} R_{k} \rightarrow 0, l \geqslant k,
$$

where $C_{l}^{0} R_{k}=R_{l}=$ sheaf of sections of $R_{l}$ the $(l-k)$ th prolongation of $R_{k}$; for $p \geqslant 1$ the $C_{l}^{p} R_{k}$ are locally free sheaves (hence the sheaves of sections of vector bundles over $M$ ) the $D$ 's are linear first order differential operators and, for $f \in \operatorname{Inf}, j_{l} f$ is just the $l$ th jet of $f$. This complex has the following properties.

(1) For $l$ large enough (in the stable range) the cohomology of $I_{l}$ is stable (independent of $l$ ), and if the original system $R_{k}$ is elliptic, so is the complex $I_{l}$. 
(2) $0 \rightarrow \operatorname{Inf} \stackrel{i_{l}}{\longrightarrow} C_{l}^{0} R_{k} \stackrel{D}{\rightarrow} C_{l}^{1} R_{k}$ is exact.

(3) There is a bracket operation on $\bigoplus_{p \geqslant 0} C_{l}^{p} R_{k}$ which makes it into a graded Lie algebra sheaf. Moreover the bracket, [, ], has the following properties.

(a) $D[u, v]=[D u, v]+(-1)^{r}[u, D v]$ for $u \in C_{l}^{r} R_{k}, v \in C_{l}^{*} R_{k}$.

(b) $[u, v]=(-1)^{r s+1}[v, u], u \in C_{l}^{r} R_{k}, v \in C_{l}^{s} R_{k}$.

(c) The Jacobi identity:

$$
(-1)^{p r}[u,[v, w]]+(-1)^{p q}[v,[w, u]]+(-1)^{q r}[w,[u, v]]=0
$$

for $u \in C_{l}^{p} R_{k}, v \in C_{l}^{q} R_{k}, w \in C_{l}^{r} R_{k}$.

We now introduce a nonlinear complex which is a finite form for the initial portion of the linear complex. Briefly, the construction goes as follows: Let $C^{\infty}(M)$ be the sheaf of germs of local $C^{\infty}$ maps of $M$ into itself, and Aut $M$ the sheaf of germs of local $C^{\infty}$ diffeomorphisms of $M$. Let $J_{l} M$ be the manifold of $l$ jets of local $C^{\infty}$ maps of $M$. The source map $\alpha_{l}: J_{l} M \rightarrow M, j_{l} f(x) \mapsto x$ and the target map $\beta_{l}: J_{l} M \rightarrow M, j_{l} f(x) \mapsto f(x)$ are submersions onto $M . J_{l} M$ has defined on it a law of composition by $j_{l} g(y) \cdot j_{l} f(x)=j_{l}(g \circ f)(x)$, where $y=$ $f(x)$. Let $\Pi_{l} M$ be the Lie groupoid of invertible elements of $J_{l} M$. The elements of $\Pi_{l} M$ are the $l$ jets of local diffeomorphisms of $M$. Let $0 \in M$ be a distinguished point, and let $\left(\Pi_{l} M\right)_{0}=\left\{\psi \in \Pi_{l} M ; \alpha_{l} \psi=0\right\}$. Then $\left(\Pi_{l} M\right)_{0}$ is a principal fibre bundle fibered by the target map $\beta_{l}$. Denote by $J_{l} M$ and $\Pi_{l} M$ the sheaf of germs of sections of $\alpha_{l}: J_{l} M \rightarrow M$ and $\alpha_{l}: \Pi_{l} M \rightarrow M$ respectively. Call $A \in \Pi_{l} M$ admissible if $\beta_{l} \circ A \in$ Aut $M$. Denote by $\Gamma_{l} M$ the subsheaf of admissible elements of $\Pi_{l} M$. ( $\Gamma_{l} M$ is not to be confused with $\Gamma(M) . \Gamma(M)$ denotes a pseudogroup on $M$.) We then have the morphism of groupoids $j_{l}$ : Aut $M \rightarrow \Gamma_{l} M$.

Now let $\Gamma(M)$ be a pseudogroup (of order $k$ ) on $M$. Let $P_{l}(\Gamma(M)) \subset \Pi_{l} M$ be the set of all elements $p \in \Pi_{l} M$ such that $p \cdot=j_{l} f$ for some $f \in \Gamma(M)$. Let $K_{l}(\Gamma(M)) \subset P_{l}(\Gamma(M))$ be the admissible elements of $P_{l}(\Gamma(M))$. Then $K_{l}(\Gamma(M)) \subset$ $\Gamma_{l} M$. Then, the following nonlinear complex may be constructed:

$$
N_{l}: 1 \rightarrow \Gamma(M) \stackrel{j_{l}}{\longrightarrow} K_{l}(\Gamma(M)) \stackrel{D}{\longrightarrow} C_{l}^{1} R_{k} \stackrel{D_{1}}{\longrightarrow} C_{l}^{2} R_{k}
$$

where $R_{k} \subset J_{k} T$ is the $k$ th order system of equations for the pseudogroup $\Gamma(M)$ $(T=T(M)=$ tangent bundle of $M)$,

$$
1 \rightarrow \Gamma(M) \stackrel{j_{l}}{\longrightarrow} K_{l}(\Gamma(M)) \stackrel{D}{\longrightarrow} C_{l}^{1} R_{k}
$$

is exact and $D_{1} D=0 . D, D_{1}$ are nonlinear differential operators and $D_{1}$ is given by $D_{1} u=D u-1 / 2[u, u]$, where $D$ is the operator of the linear complex and [,] is the bracket on that complex.

We. list the following facts. 
(1) Let $F_{t}$ be a 1-parameter family of sections of $K_{l}(\Gamma(M))$, depending differentiably on the parameter $t$, and such that $F_{0}=j_{k}$ Id; then $d F_{t} /\left.d t\right|_{t=0} \in$ $C^{\infty}\left(M, R_{l}\right)$ where $C^{\infty}\left(M, R_{l}\right)$ denotes the space of global sections of the sheaf $R_{l} \cdot\left(C^{\infty}\left(M, R_{l}\right)=C^{\infty}\left(M, R_{l}\right)=C^{\infty}\right.$ sections of the vector bundle $\left.R_{l}\right)$

(2) There exists a differentiable map $\operatorname{Exp:} C^{\infty}\left(M, R_{l}\right) \rightarrow C_{a}\left(M, K_{l}(\Gamma(M))\right)$ where $C_{a}\left(M, K_{l}(\Gamma(M))\right)$ is the space of global sections $F$ such that $\beta_{l} \circ F$ is an automorphism of $M$ where $\beta_{l}$ is the target map.

Exp has the following properties:

(a) For each $\xi \in C^{\infty}\left(M, R_{l}\right)$, the map $t \in \mathbf{R} \mapsto \operatorname{Exp} t \xi \in C_{a}\left(M, K_{l}(\Gamma(M))\right)$ is a 1-parameter subgroup of $C_{a}\left(M, K_{l}(\Gamma(M))\right)$. That is,

$$
\begin{aligned}
\operatorname{Exp}\left(t+t^{\prime}\right) \xi & =(\operatorname{Exp} t \xi) \cdot\left(\operatorname{Exp} t^{\prime} \xi\right), \\
\operatorname{Exp} 0 & =j_{l} \mathrm{Id} \\
\operatorname{Exp}(-t \xi) & =(\operatorname{Exp} t \xi)^{-1}, \\
\left.\frac{d}{d t} \operatorname{Exp} t \xi\right|_{t=0} & =\xi
\end{aligned}
$$

(b) $\beta_{l} \circ \operatorname{Exp} t \xi=\exp t(\bar{\xi})$ where $\bar{\xi}$ is the projection of $\xi$ onto $M$, i.e., $\bar{\xi}$ is a $\Gamma(M)$ vector field on $M$, and exp is the usual exponential map on $M$.

The complex $N_{l}$ is a finite form for the complex $I_{l}$. More precisely, there exists a contravariant action of $K_{l}(\Gamma(M))$ on $C_{l}^{p} R_{k}$ which we denote by Ad, such that

$$
\left.\frac{d}{d t} D F_{t}\right|_{t=t_{0}}=\operatorname{Ad} F_{t_{0}}(D \eta)
$$

where $\left\{F_{t}\right\}$ is a differentiable 1-parameter family of sections of $K_{l}(\Gamma(M))$ over an open set $U \subset M$, with $F_{t_{0}}$ admissible, $\eta=d F_{t} /\left.d t\right|_{t=t_{0}} \in C^{\infty}\left(V, R_{l}\right)$ where $V=$ $f_{t_{0}}(U)$; and

$$
\left.\frac{d}{d t} D_{1} v_{t}\right|_{t=t_{0}}=D u-\left[u, v_{t_{0}}\right]
$$

where $\left\{v_{t}\right\}$ is a differentiable 1-parameter family of sections of $C_{l}^{1} R_{k}$ over $U$ and $u=d v_{t} /\left.d t\right|_{t=t_{0}}$.

The following properties hold for the operators $D$ and $D_{1}$.

(1) $D(F \cdot G)=\operatorname{Ad} G(D F)+D G$. In particular $D(F \cdot G)=D G \Leftrightarrow F=$ $j_{k} f$, for $f \in \Gamma(M)$; and $D F^{-1}=-\operatorname{Ad} F^{-1}(D F)$.

(2) $D[\operatorname{Ad} F(u)]=\operatorname{Ad} F\left(D u-\left[D F^{-1}, u\right]\right)$.

(3) For $u \in C_{l}^{1} R_{k}$ and $F \in K_{l}(\Gamma(M))$, define $u^{F}=\operatorname{Ad} F(u)+D F$. Then we have

$$
u^{F \cdot G}=\left(u^{F}\right)^{G}, u^{\mathrm{Id}}=u, u^{F^{-1}}=\operatorname{Ad} F^{-1}(u-D F),
$$

and $D_{1}\left(u^{F}\right)=\operatorname{Ad} F\left(D_{1} u\right)$. 
The last relation shows, in particular, that $u$ satisfies the integrability condition $D_{1} u=0 \Leftrightarrow u^{F}$ satisfies the integrability condition.

Finally, we introduce one more operator as follows. Let $\left\{v_{t}\right\}$ be a differentiable $n$-parameter family of sections of $C_{l}^{1} R_{k}$ with $v_{0}=0$. Define $D_{t}: C_{l}^{p} R_{k} \rightarrow$ $C_{l}^{p+1} R_{k}$ by $D_{t} u=D u-\left[v_{t}, u\right]$. It is easily seen that $\left(D_{t}\right)^{2}=0 \Leftrightarrow D_{1} v_{t}=0$.

The differential operators $D, D, D_{1}$ are restrictions to the complexes $I_{l}, N_{l}$ of differential operators on the corresponding complexes for vector bundles [11], [25]. We have occasion to use this fact in the proof of Proposition 3.5. It is clear from the context there that we are considering $D$ as such a restriction.

We mention here in passing that Spencer's machinery has its most natural interpretation in the context of derivations of vector valued differential forms [11]. The structure of such derivations has been worked out by Henrich and Nickerson [6], [16].

2. Deformation of pseudogroup structures [5], [11], [17], [20], [21], [22]. Let $\Gamma\left(\mathbf{R}^{m}\right)$ be a transitive continuous Lie pseudogroup of order $k$ on $\mathbf{R}^{m}$. Let $U \subset M$ be an open set and let $S(U)=\left\{\varphi \mid \varphi: U \rightarrow \mathbf{R}^{m}\right.$ is a local diffeomorphism onto the domain of an element of $\left.\Gamma\left(\mathbf{R}^{m}\right)\right\}$.

$$
A(U)=\left\{(\varphi, \psi) \mid \varphi, \psi \in S(U) \text { and } \varphi \circ \psi^{-1} \in \Gamma\left(\mathrm{R}^{m}\right)\right\}
$$

If $\psi_{1}=\varphi_{2}$ we define the product $\left(\varphi_{1}, \psi_{1}\right)\left(\varphi_{2}, \psi_{2}\right)=\left(\varphi_{1}, \psi_{2}\right)$. We define an equivalence relation on $S(U)$ by $\varphi \sim \psi \Leftrightarrow(\varphi, \psi) \in A(U)$. Then $S(U)$, $A(U), S(U) / A(U)$ are presheaves on $M$. Let the associated sheaves be $S, A$ and $S / A$ and denote their spaces of sections by $H^{0}(M, S), H^{0}(M, A)$ and $H^{0}(M, S / A)$ respectively.

DEFinition 2.1. A $\Gamma\left(\mathrm{R}^{m}\right)$ structure on $M$ is an element $s \in H^{0}(M, S / A)$. A $\Gamma\left(\mathbf{R}^{m}\right)$ manifold is a pair $(M, s)$ where $M$ is a $C^{\infty}$ manifold and $s$ is a $\Gamma\left(\mathbf{R}^{m}\right)$ structure on $M$. (Integrable $G$-structures are the best known examples of pseudogroup structures [10], [19], [24] .)

Let $M_{0}=\left(M, s_{0}\right)$ be a $\Gamma\left(\mathbf{R}^{m}\right)$ structure on $M$. The local automorphisms of the structure form a pseudogroup on $M$ which we denote either by $\Gamma\left(M_{0}\right)$ or $\Gamma\left(M, s_{0}\right)$. Any pseudogroup so induced is locally equivalent to $\Gamma\left(\mathbf{R}^{m}\right)$. Hence, the pseudogroups associated to any two $\Gamma\left(\mathbf{R}^{m}\right)$ structures are locally equivalent.

In the sequel we shall be considering families of $\Gamma\left(\mathrm{R}^{m}\right)$ structures on $M$, parametrized by analytic varieties. Let $W \subset \mathrm{R}^{n}$ be an open set, and let $S$ be an analytic variety in $W$. Let $S_{r}$ be the set of regular points of $S$. (By analytic variety we mean the set of common zeros of a finite number of analytic functions.)

DEFinition 2.2. Let $S$ be an analytic variety and let $F: S \rightarrow \mathbf{R}^{k}$ be a map. 
$F$ will be called $C^{\infty}$ if $F$ is continuous and if $\left.F\right|_{S_{r}}$ is $C^{\infty}$ in the usual sense. A continuous map $F: S \rightarrow S^{\prime}$ of analytic varieties is $C^{\infty}$ if $\left.F\right|_{F^{-1}\left(S_{r}^{\prime}\right)}$ is $C^{\infty}$.

Definition 2.3. Let $U \subset M$ and $S^{\prime} \subset S$ be open sets. A $C^{\infty}$ family of coordinates of $M$ with parameter space $S$ is a $C^{\infty}$ map $\Xi: U \times S^{\prime} \rightarrow \mathrm{R}^{m}$ such that $\Xi^{s}(x)=\Xi(x, s)$ is a coordinate map for the differentiable structure on $M$ for each $s \in S^{\prime}$.

Definition 2.4. A $C^{\infty}$ family of $\Gamma\left(\mathbf{R}^{m}\right)$ structures on $M$ with parameter space $S$ is a collection $\left\{\Xi_{\alpha}\right\}$ of $C^{\infty}$ families of coordinates of $M$ such that

(1) for any $p \in M$ and any $s \in S$, there is a $\Xi_{\gamma}$ with domain $U_{\gamma} \times S^{\prime}$ and $(p, s) \in U_{\gamma} \times S^{\prime}$

(2) for each fixed $t$, the collection $\left\{\Xi_{\alpha}^{t}\right\}$ defines a $\Gamma\left(\mathbf{R}^{m}\right)$ structure on $M$.

The $\Gamma\left(\mathbf{R}^{m}\right)$ structure defined by the collection $\left\{\Xi_{\alpha}^{t}\right\}$ is labelled $M_{t}$. Hence, we obtain a $C^{\infty}$ family of $\Gamma\left(\mathbf{R}^{m}\right)$ structures on $M,\left\{M_{s}, s \in S\right\}$. Let $s_{0} \in S$ be a distinguished point; then a family $\left\{M_{s}, s \in S\right\}$ of $\Gamma\left(\mathbf{R}^{m}\right)$ structures with parameter space $S$ is called a family of deformations of the structure $M_{s_{0}}$. If $M_{0}$ is a fixed $\Gamma\left(\mathrm{R}^{m}\right)$ structure on $M$, then a family of deformations of $M_{0}$ with parameter space $S$ is a family of $\Gamma\left(\mathrm{R}^{m}\right)$ structures $\left\{M_{s}, s \in S\right\}$ such that there exists a distinguished point $s_{0} \in S$ with $M_{s_{0}}=M_{0}$. We then say that $\left\{M_{s}, s \in S\right\}$ is a $C^{\infty}$ family of deformations of $M_{0}$ over $\left(S, s_{0}\right)$.

Definition 2.5. Let $\left\{M_{s}, s \in S\right\}$ be a $C^{\infty}$ family of deformations of $M_{0}$ over $\left(S, s_{0}\right)$. Then, the family $\left\{M_{s}, s \in S\right\}$ is called complete (versal) at $s_{0}$ if for any other family $\left\{M_{t}, t \in T\right\}$ of deformations of $M_{0}$ over $\left(T, t_{0}\right)$, there exist an open neighborhood $T^{\prime} \subset T$ with $t_{0} \in T^{\prime}$, a $C^{\infty} \operatorname{map} \tau:\left(T^{\prime}, t_{0}\right) \rightarrow\left(S, s_{0}\right)$ and a collection $\left\{f^{t}, t \in T^{\prime}\right\}$ of diffeomorphisms of $M$ such that

(1) $f^{t_{0}}=\mathrm{Id}$;

(2) $f^{t}$ is a $\Gamma\left(\mathbf{R}^{m}\right)$ isomorphism between $M_{t}$ and $M_{\tau(t)}$; and

(3) for each $p \in M, f^{t}(p)$ is $C^{\infty}$ in $t$.

$\left\{M_{s}, s \in S\right\}$ is called universal if the map $\tau$ is unique.

Definition 2.6. The pseudogroup $\Gamma\left(\mathbf{R}^{m}\right)$ is called elliptic if the system of linear differential equations, $R_{k}$, associated to $\Gamma\left(\mathbf{R}^{m}\right)$ is elliptic.

From now on, we assume that $\Gamma\left(\mathbf{R}^{m}\right)$ is elliptic and that we are working in the stable range. Let $M_{0}=\left(M, s_{0}\right)$ be a $\Gamma\left(\mathbf{R}^{m}\right)$ structure on $M$, and let Inf be the sheaf of germs of $\Gamma\left(M_{0}\right)$ vector fields on $M$, i.e., Inf is the sheaf of germs of infinitesimal automorphisms of $M_{0}$. We then have the linear and nonlinear complexes

$$
\begin{gathered}
I_{l}: 0 \rightarrow \operatorname{Inf} \stackrel{j_{l}}{\longrightarrow} C_{l}^{0} R_{k}^{M_{0}} \stackrel{D}{\longrightarrow} C_{l}^{1} R_{k}^{M_{0}} \stackrel{D}{\longrightarrow} \cdots \stackrel{D}{\longrightarrow} C_{l}^{m} R_{k}^{M_{0}} \rightarrow 0, \\
N_{l}: 1 \rightarrow \Gamma\left(M_{0}\right) \stackrel{i_{l}}{\longrightarrow} K_{l}\left(\Gamma\left(M_{0}\right)\right) \stackrel{D}{\longrightarrow} C^{1} R^{M_{0}} \stackrel{D_{1}}{\longrightarrow} C_{l}^{2} R_{k}^{M_{0}}
\end{gathered}
$$


The basic existence theorem is the following

Theorem 2.1 (MAlgRANGe [15]). If $\Gamma\left(\mathbf{R}^{m}\right)$ is elliptic, the sequence

$$
1 \rightarrow \Gamma\left(M_{0}\right) \stackrel{j_{l}}{\longrightarrow} K_{l}\left(\Gamma\left(M_{0}\right)\right) \stackrel{0}{\longrightarrow} \widetilde{C}_{l}^{1} R_{k}^{M_{0}} \stackrel{D_{1}}{\longrightarrow} C_{l}^{2} R_{k}^{M_{0}}
$$

is exact, where

$$
\widetilde{C}_{l}^{1} R_{k}^{M_{0}}=\left\{u \in \mathcal{C}_{l}^{1} R_{k}^{M_{0}} \mid\left(\mathrm{Id}-\rho_{0} u\right) \in \operatorname{Aut}_{0}(\underline{T})\right\}
$$

and $\rho_{0}$ is the natural projection onto $\underline{T}^{*} \otimes \underline{T}$ and Aut ${ }_{0}(\underline{T})$ denotes the automorphisms of the sheaf $\underline{T}$. (For a vector bundle $E, \underline{E}$ denotes the sheaf of sections of $E ; O$ is the structure sheaf of $M$.

Let us examine the meaning of this theorem. Let $v$ be a global section of $\widetilde{C}_{l}^{1} R_{k}^{M_{0}}$ satisfying the integrability condition $D_{1} v=0$. Then, by the theorem of Malgrange there exist an open cover of $M$ by sets $\left\{U_{\alpha}\right\}$ and a collection $\left\{F_{\alpha}\right\}$ of sections of $K_{l}\left(\Gamma\left(M_{0}\right)\right)$ over $\left\{U_{\alpha}\right\}$ such that $D F_{\alpha}=\left.v\right|_{U_{\alpha}}$. If $U_{\alpha} \cap U_{\beta}$ is not empty, on the overlap we have $D F_{\beta}=D F_{\alpha}=\left.v\right|_{U_{\alpha} \cap U_{\beta}}$. Hence, by the exactness of the above sequence we have $F_{\alpha} \circ F_{\beta}^{-1}=j_{l} f_{\alpha \beta}$ for some $f_{\alpha \beta} \in \Gamma\left(M_{0}\right)$. Hence the collection of pairs $\left\{\left(U_{\alpha}, F_{\alpha}\right)\right\}$ defines a $\Gamma\left(\mathbf{R}^{m}\right)$ structure on $M$.

Now, let $v_{t}$ be a parametrized family of global sections of $C_{l}^{1} R_{k}^{M_{0}}$ satisfying the integrability condition $D_{1} v_{t}=0$ for each $t$, and with $v_{t_{0}}=0$. Then, for $t$ close to $t_{0}, v_{t}$ is a section of $\widetilde{C}_{l}^{1} R_{k}^{M_{0}}$ and hence we obtain a $C^{\infty}$ family $\left\{F_{t, \alpha}\right\}$ of sections of $K_{l}\left(\Gamma\left(M_{0}\right)\right)$ over $\left\{U_{\alpha}\right\}$ such that for each $t, F_{t, \alpha} \circ F_{t, \beta}^{-1}=j_{l} f_{t, \alpha \beta}$ for $f_{t, \alpha \beta} \in \Gamma\left(M_{0}\right)$. The collection of pairs $\left\{U_{\alpha}, F_{t, \alpha}\right\}$ then defines a $C^{\infty}$ deformation of the $\Gamma\left(\mathbf{R}^{m}\right)$ structure $M_{0}$. We shall now prove the converse, namely that every germ of deformations of $M_{0}$ is given by a collection of pairs $\left\{U_{\alpha}, F_{t, \alpha}\right\}$ such that for each $t$ and $U_{\alpha} \cap U_{\beta}$ nonempty, $F_{t, \alpha} \circ F_{t, \beta}^{-1}=j_{l} f_{t, \alpha \beta}$ for some $f_{t, \alpha \beta}$ $\in \Gamma\left(M_{0}\right)$.

Let $\left\{\Xi_{\alpha}\right\}$ be a family of deformations of $M_{0}$ over the parameter space $\left(S, s_{0}\right)$. Let $\Xi_{\gamma}: U_{\gamma} \times S^{\prime} \rightarrow \mathbf{R}^{m}$, and assume that $s_{0} \in S^{\prime}$. Let $U \subset U_{\gamma} \times S^{\prime}$ be the set of all $(p, s), p \in U_{\gamma}, s \in S^{\prime}$ such that $\Xi_{\gamma}(p, s) \in \Xi_{\gamma}^{0}\left(U_{\gamma}\right)$. Then $U$ is an open subset of $U_{\gamma} \times S^{\prime}$, and $U_{\gamma} \times s_{0} \subset U$. Then for each $p \in U_{\gamma}$, there exist an open neighborhood $U_{p}$ of $p$ and an open set $S_{p} \subset S^{\prime}, S_{p} \ni s_{0}$, such that the map $\varphi_{s, p}(x)=\left(\Xi_{\gamma}^{0}\right)^{-1}\left(\Xi_{\gamma}^{s}(x)\right)$ is defined for all $x \in U_{p}$ and all $s \in S_{p}$. Such a family of maps defines a family of sections of the sheaf Aut $M$ (over the open sets $U_{p}$ ) parameterized by $S$, such that $\varphi_{s_{0}, p}=\operatorname{Id}$ for each $p \in M$, and $\varphi_{s, p^{\circ}} \varphi_{s, q}^{-1}=f_{s, p q}$ $\in \Gamma\left(M_{0}\right)$ whenever this composition makes sense. If $M$ is compact, we can extract a finite subcollection $\left\{U_{p_{i}}, \varphi_{s, p_{i}}\right\}$ and this subcollection then defines a deformation of $M_{0}$. 
Let $M_{s}$ be any $\Gamma\left(\mathrm{R}^{m}\right)$ structure on $M$. If we pick a distinguished point in $M, \Gamma\left(M_{s}\right)$ gives rise to a principal fibre bundle on $M$ which we denote by $P_{l}\left(M_{s}\right)$ (see $\S 1$ ). Let us suppose now that $M_{s}$ is obtained from $M_{0}$ by the following data: an open cover $\left\{U_{\alpha}\right\}$ of $M$ and a collection $\left\{f_{\alpha}\right\}$ of local diffeomorphisms of $M$ where each $f_{\alpha}$ has domain $U_{\alpha}$ such that $f_{\alpha} \circ f_{\beta}^{-1} \in \Gamma\left(M_{0}\right)$ whenever this composition makes sense. For any $l, j_{l} f_{\alpha} \in C_{a}\left(U, \Gamma_{l} M\right)$ and the law of composition in the groupoid $\Pi_{l} M$ defines a diffeomorphism of principal fibre bundles, $j_{l} f_{\alpha}:\left.\left.P_{l}\left(M_{s}\right)\right|_{U_{\alpha}} \rightarrow P_{l}\left(M_{0}\right)\right|_{f\left(U_{\alpha}\right)}$.

Definition 2.7. The $\Gamma\left(\mathrm{R}^{m}\right)$ structures $M_{0}$ and $M_{s}$ are close if $P_{l}\left(M_{0}\right)$ and $P_{l}\left(M_{s}\right)$ are isomorphic as fibre bundles for every $l$.

Proposition 2.1. Let $M$ be compact and let $\left\{M_{s}, s \in S\right\}$ be a $C^{\infty}$ family of deformations of $M_{0}$ over $\left(S, s_{0}\right)$. Then there exists an open neighborhood $S^{\prime}$ of $s_{0}$, such that for every $s \in S^{\prime}, M_{s}$ and $M_{0}=M_{s_{0}}$ are close.

Proof. Since $M$ is compact, there exist an open $S^{\prime \prime} \subset S, s_{0} \in S^{\prime \prime}$ and a finite collection $\left\{U_{p_{i}}, \varphi_{s, p_{i}}\right\}$ where the family $\left\{U_{p_{i}}\right\}$ covers $M$, and the local diffeomorphisms $\varphi_{s, p_{i}}$ are defined for each $s \in S^{\prime \prime}$.

For each $x \in M$ and $s \in S^{\prime \prime}$, define $P_{l}^{(x, s)}=P_{l}\left(M_{s}\right)_{x}$. We first show that $P_{l}=\bigcup_{s \in S^{\prime \prime} ; x \in M} P_{l}^{(x, s)}$ is a principal fibre bundle over $M \times S^{\prime \prime}$. It clearly suffices to establish local triviality over sets of the type $U_{p_{i}} \times S^{\prime \prime}$. This is immediate since the maps $j_{l} \varphi_{s, p_{i}}$ define an isomorphism of $\left.P_{l}\left(M_{s}\right)\right|_{U_{p_{i}}}$ with $\left.P_{l}\left(M_{0}\right)\right|_{\varphi_{s, p_{i}}}\left(U_{p_{i}}\right) \cdot$

Now any point $s \in S$ has an open neighborhood which is contractible (in $S$ ) to $s$. Moreover, the contraction can be made $C^{\infty}$ at the regular points and continuous at the singular points (personal communication, John Mather). In particular, there is an open neighborhood $S^{\prime}$ of $s_{0}$ which is contractible to $s_{0}$. Then, standard methods in the theory of fibre bundles show that $P_{l}\left(M_{s}\right)$ and $P_{l}\left(M_{0}\right)$ are isomorphic for $s \in S^{\prime}$. Q.E.D.

We have actually shown more than the statement of the proposition. We have shown that for each $s \in S^{\prime}$, there exists an isomorphism $G_{s}: P_{l}\left(M_{0}\right) \rightarrow$ $P_{l}\left(M_{s}\right)$ (once a $C^{\infty}$ contraction of $S^{\prime}$ is chosen) and what is more, the family $\left\{G_{s}\right\}$ is $C^{\infty}$ in $S$.

We thus obtain a family of isomorphisms $G_{s}: P_{l}\left(M_{0}\right) \rightarrow P_{l}\left(M_{s}\right)$ for $s \in S^{\prime}$. Recall that we have a finite collection of pairs $\left\{U_{p_{i}}, \varphi_{s, p_{i}}\right\}$ on the compact manifold $M$. Define $F_{s, p_{i}}$ on $U_{p_{i}}$ by $F_{s, p_{i}}=j_{l} \varphi_{s, p_{i}} \circ G_{s}$. Then, $F_{s, p_{i}} \circ F_{s, p_{i}}^{-1}=$ $j_{l} f_{s, p_{i} p_{j}}$ where $f_{s, p_{i} p_{j}} \in \Gamma\left(M_{0}\right)$ as an easy computation shows. Hence, for a compact manifold $M$ we have shown that given a $C^{\infty}$ family $\left\{M_{s}, s \in S\right\}$ of deformations of $M_{0}$ over $\left(S, s_{0}\right)$, there exists an open neighborhood $S^{\prime}$ of $s_{0}$, such that 
$\left\{M_{s}, s \in S^{\prime}\right\}$ is given by a finite collection of pairs $\left\{U_{p_{i}}, F_{s, p_{i}}\right\}$, where the $F_{s, p_{i}}$ are sections of $K_{l}\left(\Gamma\left(M_{0}\right)\right)$ over $U_{p_{i}}$. We can now state the main theorem.

MAIN THEOREM. Let $M$ be a compact $C^{\infty}$ manifold without boundary of dimension $m$. Let $\Gamma\left(\mathbf{R}^{m}\right)$ be a continuous, transitive, elliptic Lie pseudogroup of order $k<\infty$. Let $M_{0}$ be a $\Gamma\left(\mathbf{R}^{m}\right)$ structure on $M$. Then there exists a versal germ of deformations of $M_{0}$.

In $\S 3$ we shall use Hodge theory to construct a parametrized family $\left\{v_{t}\right\}$ of global sections of $C_{l}^{1} R_{k}^{M_{0}}$ satisfying the integrability condition and with $v_{0}=$ 0 . Since for $t$ small, $v_{t}$ is a section of $\widetilde{C}_{l}^{1} R_{k}^{M_{0}}$, Malgrange's theorem then gives us a family of deformations of $M_{0}$. We finally show that this family is versal.

We have seen that given a $C^{\infty}$ family of deformations of $M_{0}$ over $\left(S, s_{0}\right)$, there exists a family of pairs $\left\{U_{p}, \varphi_{s, p}\right\}$ where $U_{p}$ is an open set containing $p$ and $\varphi_{s, p}$ is a local diffeomorphism of $U_{p}$. For each $s, \varphi_{s, p}{ }^{\circ} \varphi_{s, q}^{-1}=f_{s, p q}$ where $f_{s, p q} \in \Gamma\left(M_{0}\right)$ whenever this composition makes sense. Let us assume now that $S$ is nonsingular (i.e., $S$ is an open region in euclidean space). Let $U_{p} \cap U_{q}$ be nonempty. Then for each $x \in U_{p} \cap U_{q}$, the map $g_{p q}(s)=f_{s, p q}(x)$ as $s$ varies in an open neighborhood $S^{\prime}$ of $s_{0}$ is a $C^{\infty}$ map, $g_{p q}: S^{\prime} \rightarrow M$ with $g_{p q}\left(s_{0}\right)=x$. This map can obviously be described in the following way. Let $v \in T_{s_{0}} S$, and let $\gamma(t)$ be a $C^{\infty}$ curve in $S$ with $\gamma(0)=s_{0}$ and $d \gamma(t) /\left.d t\right|_{t=0}=v$. Let $\left\{U_{p}, \varphi_{\gamma(t), p}\right\}$ be the family of pairs with the local diffeomorphisms $\varphi_{s, p}$ restricted to $\gamma(t)$. Then, $\varphi_{\gamma(t), p} \circ \varphi_{\gamma(t), q}^{-1}=f_{\gamma(t)^{\prime}, p q}$ and for any $x \in U_{p} \cap U_{q}$, the map $f_{\gamma(t), p q}(x)$ defines a $C^{\infty}$ curve on $M$ with $f_{\gamma(0), p q}(x)=x$. Then

$$
d g_{p q}(v)=\left.\frac{d}{d t}\left(f_{\gamma(t), p q}\right)\right|_{t=0} .
$$

In this way, we obtain a vector field (keep $v$ fixed and vary $x \in U_{p} \cap U_{q}$ ) defined on $U_{p} \cap U_{q}$. Since $f_{\gamma(t), p q} \in \Gamma\left(M_{0}\right)$, the vector field $\theta_{p q}$ is a section of Inf. It is easy to check that the cohomology class of the collection $\left\{\theta_{p q}\right\}$ in $H^{1}(M, \mathrm{Inf})$ is independent of the choices made and is well defined. Hence, the map $d g_{p q}: T_{s_{0}} S \rightarrow T_{x} M$ induces a map $\rho_{M_{s}}: T_{s_{0}} \rightarrow H^{1}(M$, Inf).

DEFinition 2.8. The map $\rho_{M_{s}}: T_{s_{0}} S \rightarrow H^{1}(M$, Inf) is called the infinitesimal deformation of $M_{0}$ associated to the $C^{\infty}$ family of deformations $\left\{M_{s}: s \in S\right\}$.

Definition 2.9. The family $\left\{M_{s}: s \in S\right\}$ is called effective if $\rho_{M_{s}}: T_{s_{0}} S$ $\rightarrow H^{1}(M, \operatorname{Inf})$ is injective.

We shall show that the versal family we construct is effective when $s_{0}$ is a regular point. 
Following Spencer [23], we prove the lemma:

LEMma 2.1. With the hypotheses of the main theorem the complex $I_{l}$ is a fine resolution for Inf in the stable range.

Proof. Since $\Gamma\left(\mathbf{R}^{m}\right)$ is elliptic and since $l$ is in the stable range, the complex

$$
C_{l}^{0} R_{k}^{M_{0}} \stackrel{D}{\longrightarrow} C_{l}^{1} R_{k}^{M_{0}} \stackrel{D}{\longrightarrow} \cdots \stackrel{D}{\longrightarrow} C_{l}^{m} R_{k}^{M_{0}}
$$

is an elliptic complex. Moreover, for each $p \geqslant 0, C_{l}^{p} R_{k}^{M_{0}}$ is the sheaf of sections of a vector bundle over $M$ and hence is a fine sheaf. We have now to demonstrate the exactness of $I_{l}$. For each ${ }^{\circ}$, let $C_{l}^{p} R_{k}^{M_{0}}$ be the sheaf of sections of the vector bundle $C_{l}^{p} R_{k}^{M_{0}}$. Let $u$ represent a germ of a section of $C_{l}^{r+1} R_{k}^{M_{0}}$ over the point $x_{0} \in M$ and let $D u=0$. By the third fundamental theorem of Cartan (Goldschmidt [4]), there exists a local $\Gamma\left(\mathbf{R}^{m}\right)$ coordinate neighborhood $U$ of $x_{0}$ such that the operator $D$ is analytic in these coordinates. Choose a metric in $\left.C_{l}^{r+1} R_{k}^{M_{0}}\right|_{U}$. Now let $U_{0}, U_{1}$ be neighborhoods of $x_{0}$ such that $\bar{U}_{0} \subset U_{1}$ and $\bar{U}_{1} \subset U$. Let $\rho$ be a differentiable function with support in $U_{1}$ and which is equal to 1 on $U_{0}$. Let $u_{1}=\rho u$. Since $D$ is elliptic, there exists a local Green's operator $L_{1}$ on $U_{1}$ such that $u_{1}=\square L_{1} u_{1}$, where $\square=D^{*} D+D D^{*}$, is the Laplacian defined locally. $u_{1}=u_{0}$ on $U$ and $u_{1}=D D^{*} L_{1} u_{1}+D^{*} D L_{1} u_{1}$. Since $D u_{1}=0$ on $U_{0}$, we have $D D^{*} D L_{1} u_{1}=0$, and certainly $D^{*} D^{*} D L_{1} u_{1}=0$. Hence, we have $\square D^{*} D L_{1} u_{1}=0$, and since $\square$ is strongly elliptic, $D^{*} D L_{1} u_{1}$ is real analytic on $U_{0}$ (in the coordinates which we have chosen). Now, $D D^{*} D L_{1} u_{1}=0$ and $D^{*} D L_{1} u_{1}$ is analytic on $U_{0}$, hence there exists $\omega$, a germ over $x_{0}$ of a section of $C_{l}^{r} R_{k}^{M_{0}}$ such that $D \omega=D^{*} D L_{1} u_{1}$. Then, over some neighborhood $U^{\prime} \subset$ $U_{0}$, we have $u=D\left(D^{*} L_{1} u_{1}+\omega\right)$. Q.E.D.

3. Proof of the main theorem (following Kuranishi [12], [13]). We shall assume known the basic facts of the Hodge theory for elliptic complexes. Readers who are unfamiliar with this may refer to [13, pp. 69-78] for a brief summary.

From now on $M_{0}$ is a fixed $\Gamma\left(\mathrm{R}^{m}\right)$ structure on the compact manifold $M$, $l$ is in the stable range and $C_{l}^{*} R_{k}^{M_{0}}$ is identified with the sheaf of germs of sections of the vector bundle $C_{l}^{*} R_{k}^{M_{0}}$. Recall that $C_{l}^{0} R_{k}^{M_{0}}=R_{l}^{M_{0}}$, so that $C_{l}^{0} R_{k}^{M_{0}}=R_{l}^{M_{0}}$.

$$
I_{l}: 0 \rightarrow \operatorname{Inf} \stackrel{i_{l}}{\longrightarrow} C_{l}^{0} R_{k}^{M_{0}} \stackrel{D}{\longrightarrow} C_{l}^{1} R_{k}^{M_{0}} \stackrel{D}{\longrightarrow} \cdots \stackrel{D}{\longrightarrow} C_{l}^{m} R_{k}^{M_{0}} \rightarrow 0
$$

is a fine resolution of the sheaf $\operatorname{Inf}$. We let $C^{\infty}\left(C_{l}^{*} R_{k}^{M_{0}}\right)$ denote the space of sections of the vector bundle $\mathrm{C}_{l}^{*} R_{k}^{M_{0}}$. We introduce metrics on these vector bundles, and let $D^{*}$ be the formal adjoint of $D$ with respect to these metrics. 
We obtain the complex

$$
C^{\infty}\left(C_{l}^{0} R_{k}^{M_{0}}\right) \stackrel{D}{\longrightarrow} C^{\infty}\left(C_{l}^{1} R_{k}^{M_{0}}\right) \stackrel{D}{\longrightarrow} \cdots \stackrel{D}{\longrightarrow} C^{\infty}\left(C_{l}^{M_{0}}\right) .
$$

We let $\square=D D^{*}+D^{*} D$ be the Laplacian. We let $H^{*}$ denote the space of harmonic sections. $\Pi_{H}: C^{\infty}\left(C_{l}^{*} R_{k}^{M_{0}}\right) \rightarrow H^{*}$ is the projection. Then we have the Hodge decomposition: for $\varphi \in C^{\infty}\left(C_{l}^{*} R_{k}^{M_{0}}\right), \varphi=\Pi_{H} \varphi+\square G \varphi$, where $G$ is the Green's operator for $\square$. We let $Q=D^{*} G=G D^{*}$. Then, $\varphi=\Pi_{H} \varphi+D Q \varphi+Q D \varphi$. Also, $Q \circ Q=D^{*} \circ Q=Q \circ D^{*}=0$. $\Pi_{H} \circ Q=Q \circ \Pi_{H}=0$. We adopt the following notation. For a vector bundle $E,\left(C^{\infty}(E), q\right)$ will denote the pre-Hilbert space of global $C^{\infty}$ sections of $E$ equipped with the Sobolev $q$-norm. $\overline{\left(C^{\infty}(E), q\right)}$ will denote the completion of the space.

Now we construct a family $\varphi(s)$ of global sections of $C_{l}^{1} R_{k}^{M_{0}}$, parametrized by $s \in S$ such that $\varphi(s)$ satisfies the integrability condition for each $s \in S$ and $\varphi\left(s_{0}\right)=0$ for a distinguished point $s_{0} \in S$.

Let $\Phi=\left\{\varphi \in C^{\infty}\left(C_{l}^{1} R_{k}^{M_{0}}\right) \mid D \varphi-1 / 2[\varphi, \varphi]=0\right.$ and $\left.D^{*} \varphi=0\right\}$. Then $\square \varphi-$ $1 / 2 D^{*}[\varphi, \varphi]=0$ for all $\varphi \in \Phi .\left(\because \square \varphi-1 / 2 D^{*}[\varphi, \varphi]=D D^{*} \varphi+D^{*} D \varphi-\right.$ $1 / 2 D^{*}[\varphi, \varphi] \cdot D D^{*} \varphi=0$ since $D^{*} \varphi=0$ and $D^{*} D \varphi-1 / 2 D^{*}[\varphi, \varphi]=D^{*}(D \varphi-1 / 2[\varphi, \varphi])$ $=0$.)

Applying Green's operator $G$, we obtain $G \square \varphi-1 / 2 G D^{*}[\varphi, \varphi]=0 \Rightarrow \varphi-$ $\Pi_{H} \varphi-1 / 2 G D^{*}[\varphi, \varphi]=0$. Putting $Q=G D^{*}$, we have

$$
\begin{aligned}
\varphi-1 / 2 Q[\varphi, \varphi] & =\Pi_{H} \varphi \\
& \Rightarrow \Phi \subset \Psi=\left\{\varphi \in C^{\infty}\left(C_{l}^{1} R_{k}^{M_{0}}\right) \mid \varphi-1 / 2 Q[\varphi, \varphi] \in H^{1}\right\}
\end{aligned}
$$

Define $F: C^{\infty}\left(C_{l}^{1} R_{k}^{M_{0}}\right) \rightarrow C^{\infty}\left(C_{l}^{1} R_{k}^{M_{0}}\right)$ by $F(\varphi)=\varphi-1 / 2 Q[\varphi, \varphi] .[\varphi, \varphi]$ depends only on the 1-jet of $\varphi$ and hence is a first order differential operator (nonlinear). Then, $1 / 2 Q[\varphi, \varphi]=1 / 2 G D^{*}[\varphi, \varphi]$, and $D^{*}[\varphi, \varphi]$ is a second order (nonlinear) differential operator and hence uniformly continuous from $\left(C^{\infty}\left(C_{l}^{1} R_{k}^{M_{0}}\right), q\right)$ to $\left(C^{\infty}\left(C_{l}^{1} R_{k}^{M_{0}}\right), q-2\right)$. Since $G$ is uniformly continuous (actually bounded linear) from $\left(C^{\infty}\left(C_{l}^{1} R_{k}^{M_{0}}\right), q-2\right)$ to $\left(C^{\infty}\left(C_{l}^{1} R_{k}^{M_{0}}\right), q\right), 1 / 2 Q[\varphi, \varphi]$ is uniformly continuous from $\left(C^{\infty}\left(C_{\infty}^{1} R_{k}^{M_{0}}\right), q\right)$ to $\left(C^{\infty}\left(C_{l}^{1} R_{k}^{M_{0}}\right), q\right)$ and hence $F$ extends to a map, still denoted by $F$,

$$
F: \overline{\left(C^{\infty}\left(C_{l}^{1} R_{k}^{M_{0}}\right), q\right)} \rightarrow \overline{\left(C^{\infty}\left(C_{l}^{1} R_{k}^{M_{0}}\right), q\right)}
$$

The Fréchet derivative $d F$ of $F$ at the origin is just the identity map, and since $F=$ Identity - Quadratic, $F$ is an analytic map of Hilbert spaces. Hence, by the inverse function theorem for Banach spaces, there exist open neighborhoods $U$, $V$ of $0 ; U, V \subset\left(C^{\infty}\left(C_{l}^{1} R_{k}^{M_{0}}\right), q\right)$ such that $F: U \rightarrow V$ is invertible, i.e., $F^{-1}$ exists and $F^{-1}: V \rightarrow U$ is analytic. Let $\epsilon>0$ be sufficiently small, and let $W=$ 
$\left\{s \in H^{1} \mid\|s\|_{q}<\epsilon\right\}$. Then $F^{-1}: W \rightarrow C^{\infty}\left(C_{l}^{1} R_{k}^{M_{0}}\right)$ and $F^{-1}(s)=\varphi(s)$ with $F(\varphi(s))=s . \varphi(s)$ still satisfies the condition

$$
\square \varphi(s)-1 / 2 D^{*}[\varphi(s), \varphi(s)]=0 .
$$

$$
\begin{aligned}
& (\because \varphi(s)-1 / 2 Q[\varphi(s), \varphi(s)]=s \\
& \quad \Rightarrow \square \varphi(s)-1 / 2 \square D^{*} G[\varphi(s), \varphi(s)]=\square s=0 \quad \text { (since } s \text { is harmonic) } \\
& \quad \Rightarrow \square \varphi(s)-1 / 2 D^{*}[\varphi(s), \varphi(s)]+1 / 2 \Pi_{H} D^{*}[\varphi(s), \varphi(s)]=0 .
\end{aligned}
$$

But, $\Pi_{H} D^{*}[\varphi(s), \varphi(s)]=0 \Rightarrow \square \varphi(s)-1 / 2 D^{*}[\varphi(s), \varphi(s)]=0$.) Hence, by a well-known theorem of Douglis and Nirenberg on quasilinear elliptic operators [2], $\varphi(s)$ is $C^{\infty}$.

Now we find necessary and sufficient conditions for $\varphi(s)$ to be in $\Phi$. $(\varphi(s)$ is already in $\Psi$.) Recall, $\Phi=\left\{\varphi \in C^{\infty}\left(C_{l}^{1} R_{k}^{M_{0}}\right) \mid D \varphi-1 / 2[\varphi, \varphi]=0\right.$ and $D^{*} \varphi$ $=0$.

It is immediate that $D^{*} \varphi(s)=0$.

$$
\begin{aligned}
& (\because \varphi(s)-1 / 2 Q[\varphi(s), \varphi(s)]=s \\
& \quad \Rightarrow D^{*} \varphi(s)-1 / 2 D^{*} D^{*} G[\varphi(s), \varphi(s)]=D^{*} s=0 \quad(s \text { is harmonic }) \\
& \left.\quad \Rightarrow D^{*} \varphi(s)=0\right) .
\end{aligned}
$$

Now,

$$
\begin{aligned}
\varphi(s) & -1 / 2 Q[\varphi(s), \varphi(s)]=s \Rightarrow D \varphi(s)=1 / 2 D Q[\varphi(s), \varphi(s)] \\
& \Rightarrow D \varphi(s)-1 / 2[\varphi(s), \varphi(s)]=1 / 2 D Q[\varphi(s), \varphi(s)]-1 / 2[\varphi(s), \varphi(s)] \\
& =1 / 2 D D^{*} G[\varphi(s), \varphi(s)]-1 / 2[\varphi(s), \varphi(s)] \\
& =-1 / 2 D^{*} D G[\varphi(s), \varphi(s)]-1 / 2 \Pi_{H}[\varphi(s), \varphi(s] \\
& =-1 / 2 Q D[\varphi(s), \varphi(s)]-1 / 2 \Pi_{H}[\varphi(s), \varphi(s)] .
\end{aligned}
$$

Since the images of $Q D$ and $\Pi_{H}$ are orthogonal in the $L_{2}$-norm, $D \varphi(s)-$ $1 / 2[\varphi(s), \varphi(s)]=0 \Leftrightarrow Q D[\varphi(s), \varphi(s)]=0$ and $\Pi_{H}[\varphi(s), \varphi(s)]=0$. But we have the following

LEMMA. $\Pi_{H}[\varphi(s), \varphi(s)]=0 \Leftrightarrow Q D[\varphi(s), \varphi(s)]=0$.

PRoOF. $Q D[\varphi(s), \varphi(s)]=2 Q[D \varphi(s), \varphi(s)]=2 Q[1 / 2 D Q[\varphi(s), \varphi(s)], \varphi(s)]$. $(\because 1 / 2 D Q[\varphi(s), \varphi(s)]=D \varphi(s))$

$$
\begin{aligned}
& =Q[[\varphi(s), \varphi(s)], \varphi(s)]-Q\left[\Pi_{H}[\varphi(s), \varphi(s)], \varphi(s)\right] \\
& \quad-Q[Q D[\varphi(s), \varphi(s)], \varphi(s)] \\
& =Q[[\varphi(s), \varphi(s)], \varphi(s)]-Q[Q D[\varphi(s), \varphi(s)], \varphi(s)]
\end{aligned}
$$

$\left(\Leftrightarrow \Pi_{H}[\varphi(s), \varphi(s)]=0\right)$. 
$[[\varphi(s), \varphi(s)], \varphi(s)]=0$ by the Jacobi identity. Hence, we finally have

$$
Q D[\varphi(s), \varphi(s)]=-Q[Q D[\varphi(s), \varphi(s)], \varphi(s)] .
$$

Let $Q D[\varphi(s), \varphi(s)]=\xi(s)$; then we have $\xi(s)=-Q[\xi(s), \varphi(s)] \Rightarrow\|\xi(s)\|_{q} \leqslant$ $c\|\xi(s)\|_{q}\|\varphi(s)\|_{q}$ where $c$ is a constant independent of $\xi(s)$ and $\varphi(s)$ (this is an easy consequence of Sobolev's lemma) $\Rightarrow \xi(s)=0$ if $\|\varphi(s)\|_{q}$ is small.

This proves the lemma.

Hence, for $\|\varphi(s)\|_{q}$ sufficiently small,

$$
\varphi(s) \in \Phi \Leftrightarrow \Pi_{H}[\varphi(s), \varphi(s)]=0 .
$$

Hence, we have a family of global sections $\varphi(s)$ of $C_{l}^{1} R_{k}^{M_{0}}$ parametrized by $s$ in a small neighborhood of 0 in $H^{1} \simeq H^{1}(M$, Inf $)$, and $\varphi(0)=0 . \varphi(s)$ satisfies the integrability condition for $\|\varphi(s)\|_{q}$ small, if and only if $s$ lies in the analytic variety $S$ defined by the equation $\Pi_{H}[\varphi(s), \varphi(s)]=0$.

This completes the first part of our task. It remains to show the versality of the family $\varphi(s)$. First, we have an immediate

COROLlaRY. If $H^{2}(M$, Inf $)=0$, then $S$ is nonsingular, and is in fact an open neighborhood of zero in $H^{1}(M, \mathrm{Inf})$.

Proof. Since $H^{2} \simeq H^{2}(M$, Inf $), H^{2}(M$, Inf $)=0 \Rightarrow \Pi_{H}[\varphi, \varphi]=0$ for all $\varphi, \varphi \in C^{\infty}\left(C_{l}^{1} R_{k}^{M}\right)$. Q.E.D.

Before we can prove the versality of the family $\varphi(s)$, we need to prove the following proposition.

Proposition 3.1. Let $v_{1}$ and $v_{2}$ be global sections of $C_{l}^{1} R_{k}^{M_{0}}$ satisfying the integrability condition. Then $v_{1}$ and $v_{2}$ define equivalent $\Gamma\left(\mathbf{R}^{m}\right)$ structures $\Leftrightarrow$ there exists $F \in C_{a}\left(M, K_{l}\left(\Gamma\left(M_{0}\right)\right)\right)$ such that $\left(v_{1}\right)^{F}=\operatorname{Ad} F\left(v_{1}\right)+D F=v_{2}$.

Proof. Suppose there exists an $F$ as stated in the proposition. Let $\left\{F_{1}^{\alpha}\right\}$ be sections of $K_{l}\left(\Gamma\left(M_{0}\right)\right)$ over $\left\{U_{\alpha}\right\}$ such that $D F_{1}^{\alpha}=v_{1}$ for each $\alpha .\left\{F_{1}^{\alpha}\right\}$ exists by the theorem of Malgrange and defines a $\Gamma\left(\mathbf{R}^{m}\right)$ structure on $M$. Then $F_{1}$ and $F_{1} \cdot F$ define equivalent $\Gamma\left(\mathrm{R}^{m}\right)$ structures on $M$ (where $F_{1} \cdot F$ is the collection $\left.\left\{F_{1}^{\alpha} \cdot F\right\}\right)$ and hence the conclusion follows from the fact that $D\left(F_{1}^{\alpha} \cdot F\right)=v_{2}$ for each $\alpha$.

Suppose now that $v_{1}$ and $v_{2}$ satisfy the integrability condition and define equivalent $\Gamma\left(\mathbf{R}^{m}\right)$ structures. This means that there exist an open cover $\left\{U_{\alpha}\right\}_{\alpha \in A}$ of $M$ and a collection $\left\{F_{1}^{\alpha}\right\}_{\alpha \in A}$ of sections of $K_{l}\left(\Gamma\left(M_{0}\right)\right)\left(F_{1}^{\alpha}\right.$ being defined on $U_{\alpha}$ ) such that $D F_{1}^{\alpha}=v_{1}$ for each $\alpha$. Similarly, we can find $\left\{V_{\beta}\right\}_{\beta \in B}$ and $\left\{F_{2}^{\beta}\right\}_{\beta \in B}$ such that $D F_{2}^{\beta}=v_{2}$ for each $\beta$. Also, each $F_{1}^{\alpha}\left(F_{2}^{\beta}\right)$ covers a local diffeomorphism $f_{1}^{\alpha}\left(f_{2}^{\beta}\right)$ of $M$. 
Now let $W_{\alpha, \beta}=U_{\alpha} \cap V_{\beta}$. Then $\left\{W_{\alpha \beta}\right\}$ is an open cover for $M$, and by restriction, we obtain the collections of sections $\left\{F_{1}^{\alpha \beta}\right\}$ and $\left\{F_{2}^{\alpha \beta}\right\}$ where $D F_{1}^{\alpha \beta}=v_{1}$ and $D F_{2}^{\alpha \beta}=v_{2}$. The collection of local diffeomorphisms $\left\{f_{1}^{\alpha \beta}\right\}$ on the open cover $\left\{W_{\alpha \beta}\right\}$ defines the $\Gamma\left(\mathbf{R}^{m}\right)$ structure $M_{1}$ associated to $v_{1}$. Similarly, the collection of local diffeomorphisms $\left\{f_{2}^{\alpha \beta}\right\}$ on the open cover $\left\{W_{\alpha \beta}\right\}$ defines the $\Gamma\left(\mathbf{R}^{m}\right)$ structure $M_{2}$ associated to $v_{2}$. Since $v_{1}$ and $v_{2}$ define equivalent $\Gamma\left(\mathbf{R}^{m}\right)$ structures, there exists a global diffeomorphism $f: M \rightarrow M$ such that the collection $\left\{f_{1}^{\alpha \beta} \circ f\right\}$ defines a $\Gamma\left(\mathbf{R}^{m}\right)$ atlas for the structure $M_{2}$ associated to $v_{2}$.

Finally, we define covers for $M$ as follows:

1. Consider $f^{-1}\left(W_{\alpha \beta}\right), \alpha \in A, \beta \in B$ and let $W_{\alpha \beta \gamma \delta}=W_{\alpha \beta} \cap f^{-1}\left(W_{\gamma \delta}\right), \alpha, \gamma$ $\in A, \beta, \delta \in B$. Then the collection $\left\{W_{\alpha \beta \gamma \delta}\right\}, \alpha, \gamma \in A, \beta, \delta \in B$ covers $M$.

2. Consider $f\left(W_{\alpha \beta}\right), \alpha \in A, \beta \in B$ and let $\bar{W}_{\alpha \beta \gamma \delta}=f\left(W_{\alpha \beta}\right) \cap W_{\gamma \delta}, \alpha, \gamma \in A$, $\beta, \delta \in B$. Then, the collection $\left\{\bar{W}_{\alpha \beta \gamma \delta}\right\}, \alpha, \gamma \in A, \beta, \delta \in B$ covers $M$. It is evident that for any $\alpha, \beta, \gamma, \delta, \alpha, \gamma \in A, \beta, \delta \in B$ there exist $\alpha^{\prime}, \beta^{\prime}, \gamma^{\prime}, \delta^{\prime}$, such that $f\left(W_{\alpha \beta \gamma \delta}\right)=\bar{W}_{\alpha^{\prime} \beta^{\prime} \gamma^{\prime} \delta^{\prime}}$.

If $f\left(W_{\alpha \beta \gamma \delta}\right)=\bar{W}_{\alpha^{\prime} \beta^{\prime} \gamma^{\prime} \delta^{\prime}}$ then $f_{1}^{\alpha^{\prime} \beta^{\prime} \gamma^{\prime} \delta^{\prime}} \circ f=\varphi^{\alpha \beta \gamma \delta} \circ f_{2}^{\alpha \beta \gamma \delta}$ where $f_{1}^{\alpha^{\prime} \beta^{\prime} \gamma^{\prime} \delta^{\prime}}$ and $f_{2}^{\alpha \beta \gamma \delta}$ are restrictions of $f_{1}^{\alpha^{\prime} \beta^{\prime}}$ and $f_{2}^{\alpha \beta}$ respectively and $\varphi^{\alpha \beta \gamma \delta} \in \Gamma\left(M_{0}\right)$. Define

$$
\bar{F}^{\alpha \beta \gamma \delta}=j_{l}\left(\varphi^{\alpha \beta \gamma \delta}\right) \circ F_{2}^{\alpha \beta \gamma \delta} \text { and } F^{\alpha \beta \gamma \delta}=\left(F_{1}^{\alpha^{\prime} \beta^{\prime} \gamma^{\prime} \delta^{\prime}}\right)^{-1} \circ\left(\bar{F}_{1}^{\alpha \beta \gamma \delta}\right) \text {. }
$$

We have to show that the collection $\left\{F^{\alpha \beta \gamma \delta}\right\}$ defines an admissible global section of $K_{l}\left(\Gamma\left(M_{0}\right)\right)$. Let $U=W_{\alpha \beta \gamma \delta} \cap W_{a b c d} \neq \varnothing$. Then

$$
\begin{aligned}
& F^{\alpha \beta \gamma \delta}=F^{a b c d} \text { on } U \\
& \Leftrightarrow\left(F_{1}^{\alpha^{\prime} \beta^{\prime} \gamma^{\prime} \delta^{\prime}}\right)^{-1} \circ\left(\bar{F}^{\alpha \beta \gamma \delta}\right)=\left(F_{1}^{a^{\prime} b^{\prime} c^{\prime} d^{\prime}}\right) \circ\left(\bar{F}^{a b c d}\right) \text { on } U \\
& \Leftrightarrow\left(F_{1}^{a^{\prime} b^{\prime} c^{\prime} d^{\prime}}\right) \circ\left(F_{1}^{\alpha^{\prime} \beta^{\prime} \gamma^{\prime} \delta^{\prime}}\right)^{-1}=\left(\bar{F}^{a b c d}\right)\left(\bar{F}^{\alpha \beta \gamma \delta}\right)^{-1} \text {. } \\
& \text { Left-hand side }=j_{l}\left[\left(f_{1}^{a^{\prime} b^{\prime} c^{\prime} d^{\prime}}\right) \circ\left(f_{1}^{\alpha^{\prime} \beta^{\prime} \gamma^{\prime} \delta^{\prime}}\right)^{-1}\right] \text {. } \\
& \text { Right-hand side }=j_{l}\left[\left(\varphi^{a b c d}\right) \circ\left(f_{2}^{a b c d}\right) \circ\left(f_{2}^{\alpha \beta \gamma \delta}\right)^{-1} \circ\left(\varphi^{\alpha \beta \gamma \delta}\right)^{-1}\right] \\
& \left.=j_{l}\left[f_{1}^{a^{\prime} b^{\prime} c^{\prime} d^{\prime}}\right) \circ f \circ f^{-1} \circ\left(f_{1}^{\alpha^{\prime} \beta^{\prime} \gamma^{\prime} \delta^{\prime}}\right)^{-1}\right] \\
& =\text { left-hand side. }
\end{aligned}
$$

Hence, the collection $\left\{F^{\alpha \beta \gamma \delta}\right\}$ defines a global section. An easy check shows that this section covers the global diffeomorphism $f$. Hence the section is admissible. Q.E.D.

Let $\varphi(s)$ be the global sections of $C_{l}^{1} R_{k}^{M_{0}}$ that we constructed. The $\varphi(s)$ are integrable and in addition satisfy the condition $D^{*} \varphi(s)=0$ for each $s$. Let $\omega(t)$, $t \in T$, be any other family of global sections of $C_{l}^{1} R_{k}^{M_{0}}$ satisfying the integrability 
condition, and such that $\omega(0)=0$. By the previous proposition, we need to find an open neighborhood $T^{\prime}$ of 0 , and a family $F(t), t \in T$, such that $F(0)=$ Id and $(\omega(t))^{F(t)}=\operatorname{Ad} F(t)(\omega(t))+D F(t)=\varphi(s(t))$ where $s(t)$ means that $s$ is a $C^{\infty}$ function of $t$.

Recall first that associated to $\Gamma\left(M_{0}\right)$ we have the principal fibre bundles $P_{l}\left(M_{0}\right)$ with structure groups $G_{l}$. Let $T\left(P_{l}\left(M_{0}\right)\right)$ be the tangent bundle of $P_{l}\left(M_{0}\right)$ and let $F\left(P_{l}\left(M_{0}\right)\right) \subset T\left(P_{l}\left(M_{0}\right)\right)$ be the vertical vectors. Then we have the short exact sequence of vector bundles over $M$ [1]

$$
0 \rightarrow F\left(P_{l}\left(M_{0}\right)\right) / G_{l} \rightarrow T\left(P_{l}\left(M_{0}\right)\right) / G_{l} \rightarrow T(M) \rightarrow 0
$$

A splitting of this sequence is a connection on $P_{l}\left(M_{0}\right)$ and defines an isomorphism

$$
T\left(P_{l}\left(M_{0}\right)\right) / G_{l} \simeq F\left(P_{l}\left(M_{0}\right)\right) / G_{l} \oplus T(M)
$$

$R_{l}^{M_{0}}$,is isomorphic to $T\left(P_{l}\left(M_{0}\right)\right) / G_{l}$ and $F\left(P_{l}\left(M_{0}\right)\right) / G_{l}$ is a bundle of Lie algebras over $M$, with fibre $g_{l}$ the Lie algebra of $G_{l}$.

Let $\xi$ be a global $C^{\infty}$ section of $R_{l}^{M_{0}}$. Then, $\xi=\left(\xi_{1}, \xi_{2}\right)$ with $\xi_{1}$ a global section of $F\left(P_{l}\left(M_{0}\right)\right) / G_{l}$ and $\xi_{2}$ a vector field on $M$. Since the fibre of $F\left(P_{l}\left(M_{0}\right)\right) / G_{l}$ can be identified with $g_{l}$, the usual exponentiation in Lie groups followed by left multiplication defines an isomorphism of fibre bundles $\widetilde{e}\left(\xi_{1}\right)$ : $P_{l}\left(M_{0}\right) \rightarrow P_{l}\left(M_{0}\right)$. Now we fix a metric on $M$. For $\xi_{2}(x) \in T_{x}(M)$, consider the geodesic starting out at $x$ and with tangent vector $\xi_{2}(x)$ at $x$. Let $p$ be the end point of the geodesic at time 1 . Let $e\left(\xi_{2}\right)(x)=p$. For small $\xi, e\left(\xi_{2}\right)$ is a diffeomorphism of $M$ onto itself. Then, the connection on $P_{l}\left(M_{0}\right)$ (induced by the splitting of the short exact sequence) allows us to lift $e\left(\xi_{2}\right)$ to a fibre bundle map $\bar{e}\left(\xi_{2}\right): P_{l}\left(M_{0}\right) \rightarrow P_{l}\left(M_{0}\right)$. Define $E(\xi): P_{l}\left(M_{0}\right) \rightarrow P_{l}\left(M_{0}\right)$ as the composite, $E(\xi)=\bar{e}\left(\xi_{2}\right) \circ \widetilde{e}\left(\xi_{1}\right) . E(\xi)$ is a bundle map over the diffeomorphism $e\left(\xi_{2}\right)$. $\left.E(\xi)\right|_{P_{l}\left(M_{0}\right)_{x}}$ depends only on $\xi(x)$.

Proposition 3.2. There exists a neighborhood $U$ of zero in $C^{\infty}\left(M, C_{l}^{1} R_{k}^{M_{0}}\right)$ in the Sobolev q-norm for $q$ large enough, such that for every $v \in U$, there exists a unique $\xi(v) \in C^{\infty}\left(M, C_{l}^{0} R_{k}^{M_{0}}\right)$ such that $D^{*}\left((v)^{E(\xi(v))}\right)=0$, and $\xi$ is $C^{\infty}$ in $v$.

Proof. Recall $H^{0}=\left\{\xi \in C^{\infty}\left(C_{l}^{0} R_{k}^{M}\right) \mid D \xi=0\right\}$ and ${ }^{1} H^{0}=$ the orthogonal complement of $H^{0}$ in the $L_{2}$-norm.

Define a map $F:{ }^{1} H^{0} \times C^{\infty}\left(C_{l}^{1} R_{k}^{M 0}\right) \rightarrow{ }^{\perp} H^{0}$ by $F(\xi, v)=D^{*}\left(v^{E(\xi)}\right)=$ $D^{*}(\operatorname{Ad} E(\xi)(v))+D^{*} D(E(\xi))$. Let $G$ be the Green's operator. An easy computation shows that $F(\xi, v)=0 \Leftrightarrow G F(\xi, v)=0$. Since $E(\xi)$ depends only on the 0 -jet of $\xi, F(\xi, v)$ depends on the 2-jet of $\xi$ and the 0 -jet of $v$ and hence is a 
second order differential operator as a function of $\xi$ and a 0 -order differential operator as a function of $v$. Hence, $F$ is uniformly continuous from

$$
\left({ }^{\perp} H^{0} \times C^{\infty}\left(C_{l}^{1} R_{k}^{M_{0}}\right), q\right) \rightarrow\left({ }^{1} H^{0}, q-2\right) .
$$

And hence, $G F$ can be extended to the completions:

$$
\left.G F: C^{\infty} \overline{\left({ }^{\perp} H^{0} \times C^{\infty}\left(C_{p}^{1} R_{k}^{M_{0}}\right), q\right)} \rightarrow \overline{\left({ }^{1} H^{0}, q\right.}\right) .
$$

Consider the partial derivative $\partial(G F) /\left.\partial \xi\right|_{(0,0)}$. It is clear that the only contribution is obtained from the term $G D^{*} D(E(\xi))$ and $\partial(G F) /\left.\partial \xi\right|_{(0,0)}=G D^{*} D=$ Identity: ${ }^{\perp} H^{0} \rightarrow{ }^{\perp} H^{0}$. (We are using the fact that the Fréchet derivative of $D$ is D.)

Hence, by the implicit function theorem in Banach spaces, there exists an open neighborhood $U$ of zero in $C^{\infty}\left(C_{l}^{1} R_{k}^{M_{0}}\right)$ such that for every $v \in U$, there exists a unique $\xi(v)$ in ${ }^{1} H^{0}$ with $\xi(0)=0$ and such that $G F(\xi(v), v)=0$. Moreover, $\xi(v)$ is $C^{\infty}$ in $v$.

But $G F(\xi(v), v)=0 \Leftrightarrow F(\xi(v), v)=0$. Hence, for every $v \in U$, there exists unique $\xi(v)$ such that $D^{*}\left((v)^{E(\xi(v))}\right)=0$. Q.E.D.

It is now clear that the family $\varphi(s)$ of deformations of $M_{0}$ is versal. For let $\omega(t)$ be a family of global sections of $C_{l}^{1} R_{k}^{M_{0}}$ satisfying the integrability condition and with $\omega(0)=0$. Then, by the proposition above, if $\omega(t)$ is sufficiently small in the Sobolev $q$-norm, there exists a unique $\xi(\omega(t))$ such that

$$
D^{*}\left((\omega(t))^{E(\xi(\omega(t)))}\right)=0 .
$$

Hence, $E(\xi(\omega(t)))$ is the global family $F(t)$ that we were seeking.

We have the corollaries:

CoROllaRY 1. If $H^{1}=0$, then the $\Gamma\left(\mathbf{R}^{m}\right)$ structure $M_{0}$ is rigid.

Proof. The versal family consists only of the element $M_{0}$.

COROLlary 2. Let $\varphi(s)$ be the versal family constructed above. Then there exists a neighborhood $U$ of zero in $C^{\infty}\left(C_{l}^{1} R_{k}^{M_{0}}\right)$ in the Sobolev q-norm for $q$ large, such that if $\varphi\left(s_{0}\right) \in U$, then $\varphi(s)$ is a versal family of deformations of $\varphi\left(s_{0}\right)$ (that is, of the $\Gamma\left(\mathbf{R}^{m}\right)$ structure defined by $\varphi\left(s_{0}\right)$ ).

Proof. Let $\omega(t), t \in T$, be a family of global sections of $C_{l}^{1} R_{k}^{M_{0}}$, satisfying the integrability condition and with $\omega(0)=\varphi\left(s_{0}\right) . \omega(t)$ defines a deformation of the $\Gamma\left(\mathbf{R}^{m}\right)$ structure defined by $\varphi\left(s_{0}\right)$. Let $U$ be the open neighborhood of zero in $C^{\infty}\left(C_{l}^{1} R_{k}^{M_{0}}\right)$ constructed in the proof of Proposition 3.2, and assume that $\varphi\left(s_{0}\right) \in U$. Then for small $t, \omega(t) \in U$. Hence, for small enough $t$, there 
exists a unique $\xi(t)$ such that $D^{*}\left((\omega(t))^{E(\xi(t))}\right)=0$ and $\xi(0)=0$. Hence, $E(\xi(t))$ defines an equivalence of $\omega(t)$ ) with $\varphi(s(t))$ (in the sense of $\Gamma\left(\mathbf{R}^{m}\right)$ structures) and $E(\xi(0))=$ Identity. This proves that $\varphi(s)$ is versal for $\varphi\left(s_{0}\right)$. Q.E.D.

We want to show now that the versal family $\varphi(s)$ that we have constructed is effective at the origin. Let us recall first that we have a fine resolution for Inf:

$$
0 \rightarrow \operatorname{Inf} \stackrel{i_{l}}{\longrightarrow} C_{l}^{0} R_{k}^{M_{0}} \stackrel{D}{\longrightarrow} C_{l}^{1} R_{k}^{M_{0}} \stackrel{D}{\longrightarrow} \cdots \stackrel{D}{\longrightarrow} C_{l}^{m} R_{k}^{M_{0}} \rightarrow 0 .
$$

This sequence of sheaves gives rise to a complex

$$
C^{\infty}\left(C_{l}^{0} R_{k}^{M_{0}}\right) \stackrel{D}{\longrightarrow} C^{\infty}\left(C_{l}^{1} R_{k}^{M_{0}}\right) \stackrel{D}{\longrightarrow} \cdots \stackrel{D}{\longrightarrow} C^{\infty}\left(C_{l}^{1} R_{k}^{M_{0}}\right),
$$

and a standard theorem in the theory of sheaves asserts that $H^{p}(M, \operatorname{Inf}) \simeq p$ th cohomology of the above complex.

Now let $\left\{M_{s}, s \in S\right\}$ be a $C^{\infty}$ family of deformations of $M_{0}$, and let $\rho_{M_{s}}: T_{s_{0}} \rightarrow H^{1}(M$, Inf) be the infinitesimal deformation. (We are assuming for the moment that $S$ is nonsingular.) Let $\left\{M_{s}, s \in S\right\}$ be represented by a family $\omega(s)$ of global sections of $C_{l}^{1} R_{k}^{M_{0}}$ satisfying the integrability condition and with $\omega\left(s_{0}\right)=0$. Then, there exist an open covering $\left\{U_{\alpha}\right\}$ of $M$, and a $C^{\infty}$ family of sections $\left\{F_{s, \alpha}\right\}$ of $K_{l}\left(\Gamma\left(M_{0}\right)\right)$ such that $F_{s, \alpha} \circ F_{s, \beta}^{-1}=j_{l} f_{s, \alpha \beta}$ for $f_{s, \alpha \beta} \in \Gamma\left(\mathbf{R}^{m}\right)$. Now, let $v \in T_{s_{0}} S$ and let $\gamma(t)$ be a $C^{\infty}$ curve in $S$ starting at $s_{0}$, such that $\gamma^{\prime}(0)=$ $v$. Then, $\rho_{M_{s}}(v)$ is represented on $U_{\alpha} \cap U_{\beta}$ by the cocycle $\theta_{\alpha \beta}=d\left(f_{\gamma(t), \alpha \beta}\right) /\left.d t\right|_{t=0}$, where $f_{\gamma(t), \alpha \beta}$ is $f_{s, \alpha \beta}$ restricted to the curve $\gamma(t)$. The injection $j_{l}$ maps $\theta_{\alpha \beta}$ into

$$
\begin{aligned}
j_{l}\left(\theta_{\alpha \beta}\right) & =\left.j_{l}\left(\frac{d}{d t}\left(f_{\gamma(t), \alpha \beta}\right)\right)\right|_{t=0}=\left.\frac{d}{d t}\left(j_{l} f_{\gamma(t), \alpha \beta}\right)\right|_{t=0} \\
& =\left.\frac{d}{d t}\left(F_{\gamma(t), \alpha}\right)\right|_{t=0}-\left.\frac{d}{d t}\left(F_{\gamma(t), \beta}\right)\right|_{t=0} .
\end{aligned}
$$

It is easily seen that $\rho_{M_{s}}(v)$ is represented by the global section $\chi$ of $C_{l}^{1} R_{k}^{M_{0}}$ given by

$$
\left.\chi\right|_{U_{\alpha}}=\left.D \frac{d}{d t}\left(F_{\gamma(t), \alpha}\right)\right|_{t=0} .
$$

$\chi$ is a global section as $\left.\chi\right|_{U_{\alpha}}=\left.\chi\right|_{U_{\beta}}$ on $U_{\alpha} \cap U_{\beta}$, since

and

$$
\left.D \frac{d}{d t}\left(F_{\gamma(t), \alpha}\right)\right|_{t=0}=\left.\frac{d}{d t}\left(D F_{\gamma(t), \alpha}\right)\right|_{t=0}
$$

$$
\partial\left(F_{\gamma(t), \alpha}\right)=\mathcal{D}\left(F_{\gamma(t), \beta}\right) .
$$

From the above, it is clear then that the global section of $C_{l}^{1} R_{k}^{M_{0}}$ representing $\rho_{M_{s}}(v)$ is $d(\omega(\gamma(t))) /\left.d t\right|_{t=0}$. We can now prove

Proposition 3.3. The versal family $\{\varphi(s), s \in S\}$ of deformations of $M_{0}$ is effective at the origin if $S$ is nonsingular. 
Proof. Recall that the family was given by an analytic map $\varphi: H^{1}: \rightarrow$ $C^{\infty}\left(C_{l}^{1} R_{k}^{M}\right), s \mapsto \varphi(s)$. The discussion above merely says that if $S$ is nonsingular at zero, then $\rho_{M_{s}}$ is simply $d \varphi$. But, we saw in the proof of the main theorem that $d \varphi=$ Identity. Q.E.D.

Finally, we want to show that "equivalent" deformations have the same infinitesimal deformation.

Definition 3.1. Let $\left\{M_{s}, s \in S\right\}$ and $\left\{M_{t}, t \in T\right\}$ be $C^{\infty}$ families of deformations of $M_{0}$ over $\left(S, s_{0}\right)$ and $\left(T, t_{0}\right)$ respectively. $\left\{M_{t}, s \in S\right\}$ and $\left\{M_{t}, t \in T\right\}$ are said to be equivalent if there exist open neighborhoods $U$ and $V$ of $s_{0}$ and $t_{0}$ respectively, $U \subset S$ and $V \subset T$, an invertible $C^{\infty}$ map $\tau:\left(V, t_{0}\right) \rightarrow$ $\left(U, s_{0}\right)$ and a collection $\left\{f^{t}, t \in V\right\}$ of diffeomorphisms of $M$ such that

(1) $f^{t_{0}}=$ Identity;

(2) $f^{t}$ is a $\Gamma\left(R^{m}\right)$ isomorphism between $M_{t}$ and $M_{\tau(t)}$; and

(3) for each $p \in M, f^{t}(p)$ is $C^{\infty}$ in $t$.

Then, we have the following

Proposition 3.4. Let $\left\{M_{s}, s \in S\right\}$ and $\left\{M_{t}, t \in T\right\}$ be equivalent deformations of $M_{0}$, where $S$ and $T$ are nonsingular. Then the following diagram commutes:

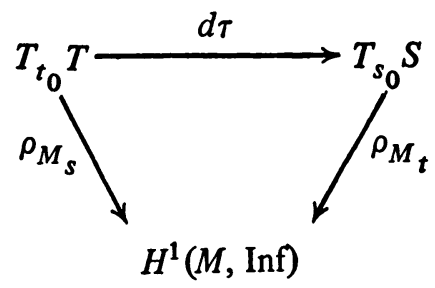

Proof. Clearly, it suffices to show the following:

Let $\gamma(u)$ be a $C^{\infty}$ curve in $T$ starting at $t_{0}$, and such that $\left.\gamma^{\prime}(u)\right|_{u=0}=v$. Then $d \tau(\gamma(u)) /\left.d u\right|_{u=0}=d \tau(v)$. Let $\omega(\gamma(u)), \varphi(\tau(\gamma(u)))$ be global sections of $C_{l}^{1} R_{k}^{M_{0}}$ defining deformations along the curves $\gamma(u)$ and $\tau(\gamma(u))$ respectively. Then we need to show

$$
\left.\left.\frac{d}{d u} \omega(\gamma(u))\right|_{u=0}=\left.\frac{d}{d u} \varphi(\tau(\gamma(u)))\right|_{u=0} \quad \text { (in } H^{1}(M, \text { Inf })\right) .
$$

Since the deformations are equivalent, there exists a parametrized family $F_{\gamma(u)}$ of admissible global sections of $K_{l}\left(\Gamma\left(M_{0}\right)\right)$ such that

$$
\text { Ad } F_{\gamma(u)}(\omega(\gamma(u)))+D F_{\gamma(u)}=\varphi(\tau(\gamma(u))) \quad \text { with } F_{\gamma(0)}=\text { Id. }
$$

We need to show that

$$
\left.\frac{d}{d u}\left(\operatorname{Ad} F_{\gamma(u)}(\omega(\gamma(u)))+D F_{\gamma(u)}\right)\right|_{u=0}=\left.\frac{d}{d u} \omega(\gamma(u))\right|_{u=0}
$$

(equal in the sense of cohomology classes in $H^{1}(M$, Inf)). But 


$$
\left.\frac{d}{d u}\left(D F_{\gamma(u)}\right)\right|_{u=0}=\left.D \frac{d}{d u} F_{\gamma(u)}\right|_{u=0}
$$

Hence $d\left(D F_{\gamma(u)}\right) /\left.d u\right|_{u=0}$ is a coboundary. Hence, it suffices to show:

But,

$$
\text { class of }\left.\frac{d}{d u}\left(\operatorname{Ad} F_{\gamma(u)}(\omega(\gamma(u)))\right)\right|_{u=0}=\text { class of }\left.\frac{d}{d u} \omega(\gamma(u))\right|_{u=0} \text {. }
$$

$$
\begin{aligned}
& \left.\frac{d}{d u}\left(\operatorname{Ad} F_{\gamma(u)}(\omega(\gamma(u)))\right)\right|_{u=0} \\
& \quad=\left(\left.\operatorname{ad}\left(\frac{d}{d u} F_{\gamma(u)}\right)\right|_{u=0}\right)(\omega(\gamma(0)))+\left.\operatorname{Ad} F_{\gamma(0)}\left(\frac{d}{d u} \omega(\gamma(u))\right)\right|_{u=0}
\end{aligned}
$$

where ad is an infinitesimal form of Ad; see Kumpera and Spencer [11]. But, $\omega(\gamma(0))=0$ and $F_{(\gamma(0))}=$ Id. Hence

$$
\left.\frac{d}{d u}\left(\operatorname{Ad} F_{\gamma(u)}(\omega(\gamma(u)))\right)\right|_{u=0}=\left.\frac{d}{d u} \omega(\gamma(u))\right|_{u=0}
$$

This proves the proposition. Q.E.D.

$A$ uniqueness theorem. We have constructed a family $\left\{M_{s}, s \in S\right\}$ of $\Gamma\left(\mathbf{R}^{m}\right)$ structures defined by a $C^{\infty}$ family $\varphi(s)$ of global sections of $C_{l}^{1} R_{k}^{M_{0}}$ satisfying the integrability condition and with $\varphi(0)=0$. This family is versal at the origin. We want to show that under suitable conditions this family is actually universal. (See Definition 2.5.)

Let $\left\{M_{t}, t \in T\right\}$ be any other family of deformations of $M_{0}$, with $M\left(t_{0}\right)=$ $M_{0}$. Let $\left\{M_{t}, t \in T\right\}$ be defined by a $C^{\infty}$ family of global sections $\omega(t)$ of $C_{l}^{1} R_{k}^{M_{0}}$ satisfying the integrability condition and with $\omega\left(t_{0}\right)=0$. Then there exist an open neighborhood $T^{\prime}$ of $t_{0}$, a $C^{\infty}$ map $\tau:\left(T^{\prime}, t_{0}\right) \rightarrow(S, 0)$, and a collection $\left\{f^{t}\right\}$ of diffeomorphisms of $M$, such that $f^{t}$ defines a $\Gamma\left(\mathbf{R}^{m}\right)$ equivalence between $M_{t}$ and $M_{\tau(t)}$, and with $f^{t_{0}}=$ Identity. We want to show that under suitable conditions the map $\tau$ is unique. (Possibly on a smaller open set $T^{\prime \prime} \subset T^{\prime}$.)

First, some notation. From now on $\left\{M_{s}, s \in S\right\}$ will always denote the versal family constructed in the proof of the main theorem, and $\varphi(s)$ the $C^{\infty}$ family of global sections of $C_{l}^{1} R_{k}^{M_{0}}$ associated to $\left\{M_{s}, s \in S\right\}$. The operators $D_{s}^{0}$ will then be defined by $D_{s}^{0}: C_{l}^{*} R_{k}^{M_{0}} \rightarrow C_{l}^{*+1} R_{k}^{M_{0}}$ by $D_{s}^{0} v=D v-[\varphi(s), v]$. Since the $\varphi(s)$ are integrable, we have $\left(D_{s}^{0}\right)^{2}=0$ and $D_{0}^{0}=D$. Hence, we obtain a $C^{\infty}$ family of sheaf complexes, (SC) $)_{l}^{s}$,

$$
(S C)_{l}^{s}=C_{l}^{0} R_{k}^{M_{0}} \stackrel{D_{s}^{0}}{\longrightarrow} C_{l}^{1} R_{k}^{M_{0}} \stackrel{D_{s}^{0}}{\longrightarrow} C_{l}^{2} R_{k}^{M_{0}} \stackrel{D_{s}^{0}}{\longrightarrow} \cdots \stackrel{D_{s}^{0}}{\longrightarrow} C_{l}^{m} R_{k}^{M_{0}}
$$

with $(S C)_{l}^{0}$ the usual linear complex we have been considering. Associated to each $\Gamma\left(\mathbf{R}^{m}\right)$ structure $M_{s}$, we have the associated sheaf $\operatorname{Inf}_{s}$ with $\operatorname{Inf}_{0}=\operatorname{Inf}$, the groupoids $K_{l}\left(\Gamma\left(M_{s}\right)\right)$ and the principal fibre bundles $P_{l}\left(M_{s}\right)$. And associated to each $\Gamma\left(\mathbf{R}^{m}\right)$ structure $M_{s}$, we have the linear complex: 


$$
C_{l}^{s}=0 \rightarrow \operatorname{Inf}_{s} \stackrel{l_{l}}{\longrightarrow} C_{l}^{0} R_{k}^{M_{s}} \stackrel{D_{s}}{\longrightarrow} C_{l}^{0} R_{k}^{M_{s}} \stackrel{D_{s}}{\longrightarrow} \cdots \stackrel{D_{s}}{\longrightarrow} C_{l}^{m} R_{k}^{M_{s}} \rightarrow 0
$$

so that $D_{0}=D=D_{0}^{0}$, and $C_{l}^{0}=(S C)_{l}^{0}=$ the usual linear complex.

Associated to the complexes $(S C)_{l}^{s}$ and $C_{l}^{s}$, we have the complexes (of $C^{\infty}$ sections of the underlying vector bundles)

$$
\begin{gathered}
\mathrm{I}_{l}^{s}: C^{\infty}\left(C_{l}^{0} R_{k}^{M_{0}}\right) \stackrel{D_{s}^{0}}{\longrightarrow} C^{\infty}\left(C_{l}^{1} R_{k}^{M_{0}}\right) \stackrel{D_{s}^{0}}{\longrightarrow} \cdots \stackrel{D_{s}^{0}}{\longrightarrow} C^{\infty}\left(C_{l}^{m} R_{k}^{M_{0}}\right), \\
\mathrm{II}_{l}^{s}: C^{\infty}\left(C_{l}^{0} R_{k}^{M_{3}}\right) \stackrel{D_{s}}{\longrightarrow} C^{\infty}\left(C_{l}^{1} R_{k}^{M_{s}}\right) \stackrel{D_{s}}{\longrightarrow} \cdots \stackrel{D_{s}}{\longrightarrow} C^{\infty}\left(C_{l}^{m_{k}} R_{k}^{M_{s}}\right) .
\end{gathered}
$$

Now let us recall that with the groupoid $\Pi_{l} M$, we can associate the principal fibre bundle $\left(\Pi_{l} M\right)_{0}$. Then, an admissible section of $\Gamma_{l} M$ defines a map of fibre bundles $\left(\Pi_{l} M\right)_{0} \rightarrow\left(\Pi_{l} M\right)_{0}$ (by the law of composition in the groupoid). An admissible section of $K_{l}\left(\Gamma\left(M_{0}\right)\right)$ then defines a map of principal fibre bundles $P_{l}\left(M_{0}\right) \rightarrow$ $P_{l}\left(M_{0}\right)$ in the same way. Let us denote by $H_{s}^{*}\left(C_{l}^{*} R_{k}^{M}\right)$ and $H_{s}^{*}\left(C_{l}^{*} R_{k}^{M}\right)$ the cohomology groups of the complexes $\mathrm{I}_{l}^{s}$ and $\mathrm{II}_{l}^{s}$ respectively. We have the following:

Proposition 3.5. Let $\left\{M_{t}, t \in T\right\}$ be a $C^{\infty}$ family of deformations of $M_{0}$. Then there exists an open neighborhood $V \subset T$ of $t_{0}$, such that there exists a $C^{\infty}$ family of admissible global sections $G(t)$ of $\Gamma_{l} M$, parametrized by $t \in V$ and with the following property. Each $G(t)$ considered as a bundle map defines an isomorphism (over the identity) $G(t): P_{l}\left(M_{0}\right) \rightarrow P_{l}\left(M_{t}\right)$ and the following diagram commutes.

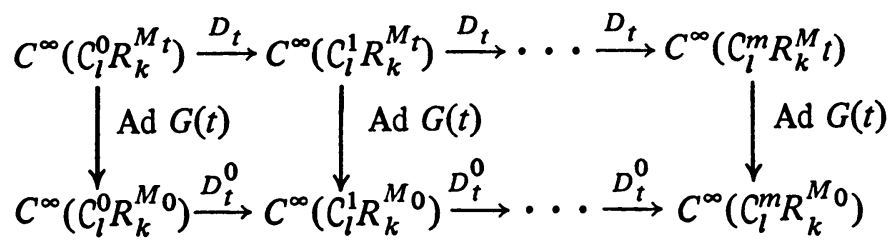

Proof. We have a covering $\left\{U_{\alpha}\right\}$ of $M$ and a collection $\left\{F_{\alpha, t}\right\}$ of local admissible sections of $K_{l}\left(\Gamma\left(M_{0}\right)\right)$ over the collection of local diffeomorphisms $\left\{f_{\alpha, t}\right\}_{t \in V}$. Over each $U_{\alpha}$, define $G_{\alpha}(t)$ by $G_{\alpha}(t)=\left(j_{l} f_{\alpha, t}\right)^{-1} \circ F_{\alpha, t}$. We show now that the collection $\left\{G_{\alpha}(t)\right\}$ defines a global section of $\Gamma_{l} M$ over the identity. Let $U_{\alpha} \cap U_{\beta}$ be nonempty and consider

$$
\begin{aligned}
G_{\alpha}(t) \circ\left(G_{\beta}(t)\right)^{-1} & =\left(j_{l} f_{\alpha, t}\right)^{-1} \circ F_{\alpha, t} \circ F_{\beta, t}^{-1} \circ j_{l} f_{\beta, t} \\
& =\left(j_{l} f_{\alpha, t}\right)^{-1} \circ j_{l}\left(f_{\alpha, t} \circ f_{\beta, t}^{-1}\right) \circ j_{l} f_{\beta, t} \\
& =j_{l}\left(f_{\alpha, t}^{-1} \circ f_{\alpha, t} \circ f_{\beta, t}^{-1} \circ f_{\beta, t}\right)=j_{l} \text { (Identity). }
\end{aligned}
$$


This proves that $G$ is globally defined over the identity. It is then clear that Ad $G(t): C^{\infty}\left(C_{l}^{*} R_{k}^{M_{t}}\right) \rightarrow C^{\infty}\left(C_{l}^{*} R_{k}^{M_{0}}\right)$ is an isomorphism. To show commutativity of the diagram, we have to show that $\operatorname{Ad} G(D \xi)=D_{t}^{0} \operatorname{Ad} G(\xi)$, for $\xi \in$ $C^{\infty}\left(C_{l}^{*} R_{k}^{M}{ }^{t}\right)$. But $D_{t}^{0} \operatorname{Ad} G \xi=D(\operatorname{Ad} G(\xi))-[D F, \operatorname{Ad} G(\xi)]$ (where by $D F$, we mean the global section of $C_{l}^{1} R_{k}^{M_{0}}$ defined by the collection $\left.\left\{D F_{\alpha}\right\}\right)$

$$
\begin{aligned}
& =\operatorname{Ad} G(D \xi)-\operatorname{Ad} G\left[D G^{-1}, \xi\right]-[D F, \operatorname{Ad} G(\xi)] \\
& =\operatorname{Ad} G(D \xi)-\left[\operatorname{Ad} G\left(D G^{-1}\right), \operatorname{Ad} G(\xi)\right]-[D F, \operatorname{Ad} G(\xi)] \\
& =\operatorname{Ad} G(D \xi)+[D G, \operatorname{Ad} G(\xi)]-[D F, \operatorname{Ad} G(\xi)] .
\end{aligned}
$$

But $D G=D F$. Hence $\operatorname{Ad} G(D \xi)=D_{t}^{0} \operatorname{Ad} G(\xi)$. Q.E.D.

COROLlaRY. If $\left\{M_{t}, t \in T\right\}$ is a family of deformations of $M_{0}$, then $\operatorname{dim} H^{p}\left(M, \operatorname{Inf}_{t}\right)$ is an upper semicontinuous function (of $\left.t\right)$.

Proof. Proposition 3.5 implies that $H^{*}\left(C_{l}^{*} R_{k}^{M}{ }^{0}\right) \simeq H_{t}^{*}\left(C_{l}^{*} R_{k}^{M t}\right) \simeq H^{*}\left(M, \operatorname{Inf}_{t}\right)$ where the second isomorphism is obtained from the fine resolution of $\operatorname{Inf}_{t}$. Now, since $M$ is compact, for small $t$, the complex (SC) ${ }_{l}^{t}$ is an elliptic complex, since it is a small deformation of the elliptic complex $(S C)_{l}^{0}$ which is the usual linear complex. Then, by a well-known theorem on elliptic complexes, $\operatorname{dim} H_{t}^{*}\left(C_{l}^{*} R_{k}^{M_{0}}\right)$ is an upper semicontinuous function. This proves our contention. Q.E.D.

Our uniqueness theorem follows from Theorem II (also see [14]).

THEOREM II. Let $\left\{M_{s}, s \in S\right\}$ be the versal family of deformations of $M_{0}$ constructed in the main theorem. Let $\operatorname{dim} H^{0}\left(M, \operatorname{Inf}_{s}\right)$ be constant in an open neighborhood of 0 in $S$. Then, there exist an open neighborhood $U$ of the identity in $C_{a}\left(M, K_{l}\left(\Gamma\left(M_{0}\right)\right)\right)$ (where $C_{a}\left(M, K_{l}\left(\Gamma\left(M_{0}\right)\right)\right)$ is the space of global admissible sections of $K_{l}\left(\Gamma\left(M_{0}\right)\right)$ equipped with Sobolev q-norm for large $q$ ), and an open neighborhood $V$ of 0 in $S$ such that every $F \in U$ can be expressed as $F=$ $\operatorname{Exp}\left(\operatorname{Ad} G(s)\left(\xi_{s}\right)\right) \circ E\left(\eta_{s}\right)$, where $\xi_{s} \in H^{0}\left(M, \operatorname{Inf}_{s}\right)=H_{s}^{0}$, and $\eta_{s} \in{ }^{\perp} H^{0}$ for each $s \in S$ and $G(s)$ is the map of Proposition 3.5. The map $E(\eta)$ is the one defined above, and the map $\operatorname{Exp}(\xi)$ is the one defined in $\S 1$ frozen at time 1 .

Proof. We prove this theorem in two steps.

Step 1. We first show that $E(\xi)$ sweeps out a small neighborhood, $W$, of the identity in $C_{a}\left(M, H_{l}\left(\Gamma\left(M_{0}\right)\right)\right)$ as $\xi$ varies in a small neighborhood $W^{\prime}$ of zero in $C^{\infty}\left(C_{l}^{0} R_{k}^{M}\right), W$ and $W^{\prime}$ being open in the Sobolev $q$-norm topology for large $q$. We know that $E(\xi)=\bar{e}\left(\xi_{2}\right) \circ \widetilde{e}\left(\xi_{1}\right)$ and since $\widetilde{e}\left(\xi_{1}\right)$ is defined by the usual exponential in Lie groups, it suffices to show that $e\left(\xi_{2}\right)$ sweeps out a small neighborhood of the identity in $\operatorname{Diff}(M)(\operatorname{Diff}(M)=$ Diffeomorphisms of $M)$.

We do this as follows: Cover $M$ by a finite number $\left\{U_{\alpha}\right\}$ of normal neighborhoods (for the metric defining $e\left(\xi_{2}\right)$ ). Choose open sets $V_{\alpha}$ such that $\bar{V}_{\alpha} \subset U_{\alpha}$ 
and $\left\{V_{\alpha}\right\}$ covers $M$. This is always possible. Consider all $f \in \operatorname{Diff}(M)$ such that $f\left(V_{\alpha}\right)$ $\subset U_{\alpha}$, for each $\alpha$. Denote the set of all such diffeomorphisms by $\operatorname{Sm} \operatorname{Diff}(M)$. It is clear that if $f \in \operatorname{Diff}(M)$ is sufficiently close to the identity in the $C_{0}$ topology, then $f$ $\in \operatorname{Sm} \operatorname{Diff}(M)$. Hence, $\operatorname{Sm} \operatorname{Diff}(M)$ contains an open neighborhood of the identity in the $C_{0}$ topology. Call this neighborhood $W$. For any $f \in W$, join $x$ and $f(x)$ by a unique geodesic. Take the tangent to this at $x$ and call it $\xi_{x}$. Then the vector field $\xi$ so defined has the property that $e(\xi)=f$. Hence, there exists a neighborhood of the zero section $W^{\prime}$ in the $C_{0}$ topology in $C^{\infty}(M, T(M))$ such that $\xi \in W^{\prime}, e(\xi) \in W$, and for every $f \in W$, there exists $\xi \in W^{\prime}$ such that $e(\xi)=f$. Now Sobolev's lemma implies that for $q$ large enough, $W$ and $W^{\prime}$ are open in the $q$-norm topology. This proves our contention.

Step 2. Associated to each $M_{s}$, we have the principal fibre bundles $P_{l}\left(M_{s}\right)$ and local bundle maps $F_{\alpha, s}:\left.\left.P_{l}\left(M_{0}\right)\right|_{U_{\alpha}} \rightarrow P_{l}\left(M_{0}\right)\right|_{U(s)}$. We defined global bundle isomorphisms $G(s), G(s): P_{l}\left(M_{0}^{\alpha}\right) \rightarrow P_{l}\left(M_{s}\right)$, and Proposition 3.5 implies that Ad $G(s)$ defines an isomorphism between $H_{s}^{0}$ and $H_{s}^{0}\left(C_{l}^{*} R_{k}^{M_{0}}\right)$. Since $\operatorname{dim} H_{s}^{0}$ is locally constant, $\operatorname{dim} H_{s}^{0}\left(\mathrm{C}_{l}^{*} R_{k}^{M_{0}}\right)$ is locally constant. Let us denote $H_{s}^{0}\left(C_{l}^{*} R_{k}^{M_{0}}\right)$ by $H_{s}^{0}$ from now on. Then we obtain a $C^{\infty}$ family of complements of ${ }^{\perp} H^{0}$ in $C^{\infty}\left(C_{l}^{0} R_{k}^{M_{0}}\right)$. That is, we have $C^{\infty}\left(C_{l}^{0} R_{k}^{M_{0}}\right) \simeq H_{s}^{0} \oplus^{\perp} H^{0}$.

For each $s$, define the map $Q_{s}$ :

$$
Q_{s}: H_{s}^{0} \oplus{ }^{\perp} H^{0} \rightarrow C^{\infty}\left(C_{l}^{0} R_{k}^{M_{0}}\right) \text { by } Q_{s}\left(\chi_{s}, \eta\right)=\varphi
$$

where $\varphi$ is the element which makes the following diagram commutative

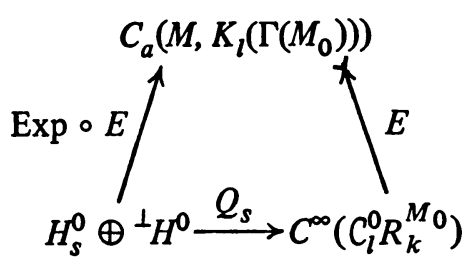

That is $\operatorname{Exp}\left(\chi_{s}\right) \circ E(\eta)=E(\varphi) . Q_{s}$ is continuously differentiable in a neighborhood of zero (in Sobolev $q$-norm) and an easy computation shows that $\left.d Q_{0}\right|_{(0,0)}$ $=$ Identity. We also note that $Q_{s}$ has the following property. Let $\left\{x_{n}\right\}$ be a Cauchy sequence in $H_{s}^{0} \oplus^{\perp} H^{0}$ (in the Sobolev $q$-norm). Suppose $\left\{Q_{s}\left(x_{n}\right)\right\}$ is

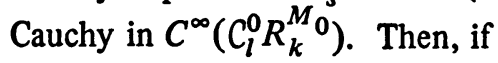

$$
\begin{aligned}
\left\{x_{n}\right\} & \rightarrow x \in \overline{\left(H_{s}^{0} \oplus^{1} H^{0}, q\right)}-\left(H_{s}^{0} \oplus{ }^{\perp} H^{0}, q\right), \text { then } \\
\left\{Q_{s}\left(x_{n}\right)\right\} & \rightarrow y \in \overline{\left(C^{\infty}\left(C_{l}^{0} R_{k}^{M_{0}}\right), q\right)}-\left(C^{\infty}\left(C_{l}^{0} R_{k}^{M_{0}}\right), q\right) .
\end{aligned}
$$

We need the following trivial generalization of the inverse function theorem for Banach spaces.

Proposition. Let $X$ and $Y$ be normed linear spaces and let $\bar{X}$ and $\bar{Y}$ be 
their completions. Let $f: X \rightarrow Y$ be continuously differentiable in a neighborhood of $0 \in X$, and let $\left.d f\right|_{0}$ be a toplinear isomorphism. In addition suppose $f$ satisfies the following property: $\left\{x_{n}\right\}$ is a Cauchy sequence in $X$ converging to $x \in \bar{X}-X$ and $\left\{f\left(x_{n}\right)\right\}$ is Cauchy in $Y \Rightarrow\left\{f\left(x_{n}\right)\right\}$ converges to $y \in \bar{Y}-Y$. Then, $f$ is invertible in a neighborhood of zero.

Applying this proposition, we see that there exists an open neighborhood $U^{\prime}$ of zero in $C^{\infty}\left(C_{l}^{0} R_{k}^{M_{0}}\right)$ such that for every $\varphi \in U^{\prime}$, there exist a $\chi_{s} \in H_{s}^{0}$ and a unique $\eta_{s} \in W$, where $W \subset{ }^{\perp} H^{0}$ is sufficiently small in the Sobolev $q$-norm such that $E(\varphi)=\operatorname{Exp}\left(\chi_{s}\right) \circ E\left(\eta_{s}\right)$. Then, Step 1 in our proof implies that if $F$ is small enough $F=\operatorname{Exp}\left(\chi_{s}\right) \circ E\left(\eta_{s}\right)$. Define $\xi_{s}=\operatorname{Ad} G(s)^{-1}\left(\chi_{s}\right)$. This proves our theorem. Q.E.D.

COROLlary 1. Let $\left\{M_{s}, s \in S\right\}$ be the versal family constructed in the main theorem. Let $\operatorname{dim} H^{0}\left(M, \mathrm{Inf}_{s}\right)$ be constant in a neighborhood of $0 \in S$. Then, there exist an open neighborhood $U$ of the identity in $C^{\infty}\left(M, K_{l}\left(\Gamma\left(M_{0}\right)\right)\right)$ ( $U$ open in the Sobolev q-norm for q-large) and an open neighborhood $V$ of $0 \in$ $S$ such that for any $F \in U$, and $s_{1}, s_{2} \in V, F$ does not define an equivalence of the $\Gamma\left(\mathbf{R}^{m}\right)$ structures $M_{s_{1}}$ and $M_{s_{2}}$.

Proof. Let $\left\{U_{\alpha}\right\}$ be an open covering for $M$ and let $M_{s_{1}}$ and $M_{s_{2}}$ be represented by the collections of local admissible sections of $K_{l}\left(\Gamma\left(M_{0}\right)\right),\left\{F_{\alpha, s_{1}}\right\}$ and $\left\{F_{\alpha, s_{2}}\right\}$. If $M_{s_{1}}$ and $M_{s_{2}}$ are equivalent, then there exists a global admissible section $F$ of $K_{l}\left(\Gamma\left(M_{0}\right)\right)$ such that for each $\alpha, F_{\alpha, s_{1}} \circ F=F_{\alpha, s_{2}}$. Let $U$ be the open neighborhood of the identity in $C_{a}\left(M, K_{l}\left(\Gamma\left(M_{0}\right)\right)\right)$ constructed in the last theorem. Then, $F=$ $\operatorname{Exp}\left(\operatorname{Ad} G\left(s_{1}\right)\left(\xi_{s_{1}}\right)\right) \circ E(\eta)$ for $\xi_{s_{1}} \in H_{s_{1}}^{0}$ and $\eta \in W \subset{ }^{\perp} H^{0}$. Then, we have

$$
\begin{aligned}
F_{\alpha, s_{1}} \circ F & =F_{\alpha, s_{1}} \circ \operatorname{Exp}\left(\operatorname{Ad} G\left(s_{1}\right)\left(\xi_{1}\right)\right) \circ E(\eta) \\
& =F_{\alpha, s_{1}} \circ G\left(s_{1}\right)^{-1} \circ \operatorname{Exp} \xi_{s_{1}} \circ G\left(s_{1}\right) \circ E(\eta) .
\end{aligned}
$$

And locally, we have

$$
\begin{aligned}
F_{\alpha, s_{1}} \circ F_{\alpha, s_{1}}^{-1} \circ j_{l} f_{\alpha, s_{1}} \circ \operatorname{Exp} \xi_{s_{1}} \circ\left(j_{l} f_{\alpha, s_{1}}\right)^{-1} \circ F_{\alpha, s_{1}} \circ E(\eta) \\
=j_{l} f_{\alpha, s_{1}} \circ \operatorname{Exp} \xi_{s_{1}} \circ\left(j_{l} f_{\alpha, s_{1}}\right)^{-1} \circ F_{\alpha, s_{1}} \circ E(\eta) .
\end{aligned}
$$

But $\xi_{s_{1}}$ is a $\Gamma\left(M_{s_{1}}\right)$ vector field, and hence

$$
\begin{aligned}
\operatorname{Exp} \xi_{s_{1}} & =j_{l} \varphi_{s_{1}} \quad \text { where } \varphi_{s_{1}} \text { is in } \Gamma\left(M_{s_{1}}\right) \\
& \Rightarrow j_{l} f_{\alpha, s_{1}} \circ \operatorname{Exp} \xi_{s_{1}} \circ\left(j_{l} f_{\alpha, s_{1}}\right)^{-1}=j_{l} \varphi, \varphi \in \Gamma\left(M_{0}\right) \\
& \Rightarrow j_{l} \varphi \circ F_{\alpha, s_{1}} \circ E(\eta)=F_{\alpha, s_{2}} .
\end{aligned}
$$

Hence, 


$$
D\left(j_{l} \varphi \circ F_{\alpha, s_{1}} \circ E(\eta)\right)=D\left(F_{\alpha, s_{2}}\right) \therefore D\left(F_{\alpha, s_{1}} \circ E(\eta)\right)=D\left(F_{\alpha, s_{2}}\right)
$$

i.e., $\operatorname{Ad}(E(\eta))\left(D F_{\alpha, s_{1}}\right)+D(E(\eta))^{-1}=D\left(F_{\alpha, s_{2}}\right)$ and since $D^{*} D\left(F_{\alpha, s_{2}}\right)=0\left(M_{s_{2}}\right.$ is in our versal family) we have

$$
D^{*}\left(\operatorname{Ad}(E(\eta)) \circ D F_{\alpha, s_{1}}+D(E(\eta))^{-1}\right)=0 .
$$

But, $D^{*} D F_{\alpha, s_{1}}=0$ and hence by Proposition 3.2, $\eta=0 \Rightarrow M_{s_{1}}=M_{s_{2}}$ and $s_{1}=s_{2}$. Q.E.D.

CoRollary 2. If $\operatorname{dim} H^{0}\left(M, \operatorname{Inf}_{s}\right)$ is constant in a neighborhood of $0 \in S$, then the versal family $\left\{M_{s}, s \in S\right\}$ is universal.

Proof. If not, there must exist a family $\left\{M_{t}, t \in T\right\}$ of deformations of $M_{0}$ and maps $\tau$ and $\tau^{\prime}: U \rightarrow S, U$ open in $T$ such that $M_{t}$ is $\Gamma\left(\mathrm{R}^{m}\right)$ equivalent to $M_{\tau(t)}$ and $M_{\tau^{\prime}(t)}$. This implies that $M_{\tau(t)}$ and $M_{\tau^{\prime}(t)}$ are $\Gamma\left(\mathrm{R}^{m}\right)$ equivalent. This contradicts Corollary 1. (In the above, we have assumed that we are working in small enough neighborhoods of zero in S.) Q.E.D.

\section{BIBLIOGRAPHY}

1. M. F. Atiyah, Complex analytic connections in fibre bundles, 'Trans. Amer. Math. Soc. 85 (1957), 181-207. MR 19, 172.

2. A. Douglis and L. Nirenberg, Interior estimates for elliptic systems of partial differential equations, Comm. Pure Appl. Math. 8 (1955), 503-538. MR 17, 743.

3. H. Goldschmidt, Existence theorems for analytic linear partial differential equations, Ann. of Math. (2) 86 (1967), 246-270. MR 36 \#2933.

4. - Sur la structure des équations de Lie: II, Équations formellement transitives, J. Differential Geometry 7 (1972), 67-95.

5. Victor Guillemin and Shlomo Sternberg, Deformation theory of pseudogroup struc. tures, Mem. Amer. Math. Soc. No. 64 (1966). MR 35 \#2302.

6. C. J. Henrich, Derivations on an arbitrary vector bundle, Trans. Amer. Math. Soc. 109 (1963), 411-419. MR 27 \#5195.

7. K. Kodaira and D. C. Spencer, On deformations of complex analytic structures. I, II, Ann. of Math. (2) 67 (1958), 328-460. MR 22 \#3009.

8. - On deformations of complex analytic structures. III. Stability theorems for complex structures, Ann. of Math. (2) 71 (1960), 43-76. MR 22 \#5991.

9. K. Kodaira, L. Nirenberg and D. C. Spencer, On the existence of deformations of complex analytic structures, Ann. of Math. (2) 68 (1958), 450-459. MR 22 \#3012.

10. K. Kodaira and D. C. Spencer, Multifoliate structures, Ann. of Math. (2) 74 (1961), 52-100. MR 26 \#5595.

11. A. Kumpera and D. C. Spencer, Lie equations. I: General theory, Ann. of Math. Studies, vol. 73, Princeton Univ. Press, Princeton, N. J., 1972.

12. M. Kuranishi, New proof for the existence of locally complete families of complex structures, Proc. Conf. Complex Analysis (Minneapolis, 1964), Springer-Verlag, Berlin, 1965, pp. 142-154. MR 31 \#768.

13. - Deformations of compact complex manifolds, University of Montreal Press, 1971.

14. - A note on families of complex structures, Global Analysis (Papers in Honor of K. Kodaira), Univ. Tokyo Press, Tokyo; Princeton Univ. Press, Princeton, N. J., 1969, pp. 309-313. MR $40 \# 8090$.

15. B. Malgrange, Equations de Lie, J. Differential Geometry 7 (1972), 117-141. 
16. H. K. Nickerson, On differential operators and connections, Trans. Amer. Math. Soc. 99 (1961), 509-539. MR 28 \#5411.

17. Ngô van Quê, Nonabelian Spencer cohomology and deformation theory, J. Differential Geometry 3 (1969), 165-211. MR 42 \#1164.

18. D. G. Quillen, Formal properties of overdetermined systems of linear partial differential equations, Thesis, Harvard University, 1964.

19. I. M. Singer and S. Sternberg, The infinite groups of Lie and Cartan. I, J. Analyse Math. 15 (1965), 1-114. MR 36 \#11.

20. D. C. Spencer, Deformations of structures on manifolds defined by transitive continuous pseudogroups. I, II, Ann. of Math. (2) 76 (1962), 306-445. MR 27 \#6287a, b.

21. , Deformation of structures on manifolds defined by transitive, continuous pseudogroups. III. Structures defined by elliptic pseudogroups, Ann. of Math. (2) 81 (1965), 389-450. MR 31 \#4052.

22. - On deformation of pseudogroup structures, Global Analysis (Papers in Honor of K. Kodaira), Univ. Tokyo Press, Tokyo; Princeton Univ. Press, Princeton, N. J., 1969, pp. 367-395. MR 41 \#4591.

23. - Overdetermined systems of linear partial differential equations, Bull. Amer. Math. Soc. 75 (1969), 179-239. MR 39 \#3533.

24. Shlomo Sternberg, Lectures on differential geometry, Prentice-Hall, Englewood Cliffs, N. J., 1964. MR 33 \#1797.

25. W. J. Sweeney, The D-Neumann problem, Acta Math. 120 (1968), 223-277. MR 37 \#2250.

DEPARTMENT OF MATHEMATICS, THE JOHNS HOPKINS UNIVERSITY, BALTIMORE, MARYLAND 21218

DEPARTMENT OF MATHEMATICS, INDIANA UNIVERSITY, BLOOMINGTON, INDIANA 47401 (Current address) 